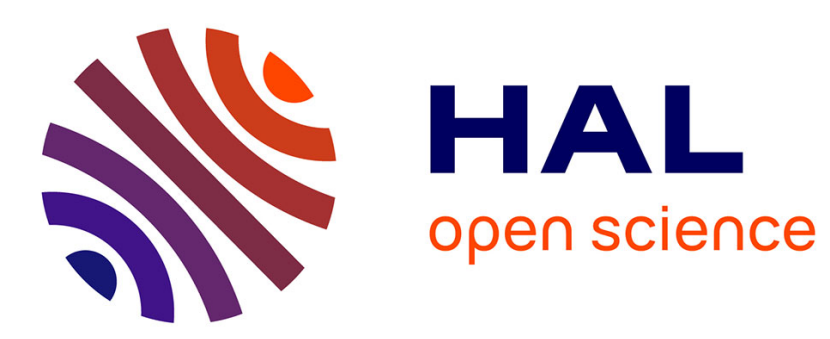

\title{
Convergence of generalized volume averaging method on a convection-diffusion problem: a spectral perspective
}

\author{
Charles Pierre, Franck Plouraboué, Michel Quintard
}

\section{To cite this version:}

Charles Pierre, Franck Plouraboué, Michel Quintard. Convergence of generalized volume averaging method on a convection-diffusion problem : a spectral perspective. SIAM Journal on Applied Mathematics, 2006, vol. 66 (no1), pp. 122-152. hal-00158286

\section{HAL Id: hal-00158286 https://hal.science/hal-00158286}

Submitted on 4 Jul 2007

HAL is a multi-disciplinary open access archive for the deposit and dissemination of scientific research documents, whether they are published or not. The documents may come from teaching and research institutions in France or abroad, or from public or private research centers.
L'archive ouverte pluridisciplinaire HAL, est destinée au dépôt et à la diffusion de documents scientifiques de niveau recherche, publiés ou non, émanant des établissements d'enseignement et de recherche français ou étrangers, des laboratoires publics ou privés. 


\title{
CONVERGENCE OF GENERALIZED VOLUME AVERAGING METHOD ON A CONVECTION-DIFFUSION PROBLEM : A SPECTRAL PERSPECTIVE
}

\author{
C. PIERRE ${ }^{*}$, F.PLOURABOUÉ $^{\dagger}$, AND M. QUINTARD ${ }^{\dagger}$
}

\begin{abstract}
This paper proposes a thorough investigation of the convergence of the volume averaging method described in [36] as applied to convection-diffusion problems inside a cylinder. A spectral description of volume averaging brings to the fore new perspectives about the mathematical analysis of those approximations. This spectral point of view is complementary with the Liapounov-Schmidt reduction technique and provides a precise framework for investigating convergence. It is shown for convection-diffusion inside a cylinder that the spectral convergence of the volume averaged description depends on the chosen averaging operator, as well as on the boundary conditions. A remarkable result states that only part of the eigenmodes among the infinite discrete spectrum of the full solution can be captured by averaging methods. This leads to a general convergence theorem (which was already examined with the use of the centre manifold theorem [22] and investigated with Liapounov-Schmidt reduction techniques [11] in similar contexts). Moreover, a necessary and sufficient condition for an eigenvalue to be captured is given. We then investigate specific averaging operators, the convergence of which is found to be exponential.
\end{abstract}

Key words. Volume averaging, homogenization, convection, diffusion, Sturm-Liouville, spectral theory, Picard's successive approximation method, spectral methods

\section{AMS subject classifications.}

1. Introduction. Volume averaging techniques are widely used to model transport problems for which decoupled or separated scales can be identified. The first part of this introduction deals with the potential interest of volume averaging for convection-diffusion problems in different applications. In the second part we discuss the interest and the specificity of volume averaging as compared to other homogenization methods. This general discussion is developed in the paper in a specific case suitable for mathematical treatment : the problem of convection-diffusion in a circular tube.

Convection-diffusion inside a tube would seem to be a simple mathematical problem. It turns out that it is a non-trivial problem, well-known in the history of applied mathematics. Starting from Graetz [16] and Lévêque [19] in the stationary case, it has more lately interested Taylor [35] and Aris [1] in the context of its transient non-stationary asymptotic behaviour. These seminal works have inspired many others, some of which are discussed in the second part of the introduction when discussing the methodological point of view.

Many research areas such as chemical engineering, bio-mechanics, porous media, are interested by variants of such a simple generic convection-diffusion problem. For example, when the considered problem involves many tubes inside which convection occurs (such as heat exchangers, or microvascular beds), transport equations have been sought in terms of cross-section averaged fields [7, $18,24,38,23]$. Recently, the design and optimisation of micro heater exchangers has stimulated the search for averaged equations governing averaged temperature either at the tube scale, or at the scale of the whole exchanger [38, 23]. In the context of heat exchange in biological tissues, averaged descriptions have remained very useful models [25] since the pioneering Pennes's model $[27,2,3]$. These investigations suggest that averaged temperature associated with "compartmental" domains such as tissues and blood flow in vessels are interesting quantities to consider in order to model heat exchanges inside bodies. In these cases it is crucial to understand how the micro-scale

\footnotetext{
*Université de Nantes Laboratoire de mathématiques Jean Leray UMR CNRS No.6629

${ }^{\dagger}$ IMFT UMR CNRS-INPT/UPS No.5502 Av. du Professeur Camille Soula 31400 Toulouse France
} 
flow may be approximated by averaged models because, even if possible, a detailed description of the full stationary problem at the local scale of each tube is not of great interest. In the context of these applications, averaged models have proved to be useful and interesting for applied concerns. Nevertheless, even if the description of averaged quantities is useful in practise for obvious operational reasons, there are still numerous questions concerning the validity and the quality of the approximation given by these ad-hoc models. As a matter of fact, even if the model predictions could be in retrospect tested numerically, it is always interesting to better understand what their mathematical foundations are. This allows one to better understand their limits and their possible extensions. In this paper, we investigate the model of stationary convection-diffusion inside a tube. This study shows that, in this particular context, an averaged description can only capture large scale features of the exact solution, the convergence of which can be made as precise as necessary.

From a methodological point of view, spatial averaging is at first used as an operational definition of macro-scale quantities. From this, macro-scale equations may be derived, and the reader is referred to the paper [12] for a review of the different perspectives and points of view. For example, macro-scale equations are introduced by many authors from extensive use of irreversible thermodynamics [17] (this approach is also often called mixture theory). In this paper, we are interested in methods that provide a direct, deterministic link, through some mapping variables, between the micro-scale and the macro-scale fields. Such a method has been applied to determine macro-scale transport equations for porous media applications, as illustrated in [36] whilst concomitantly a very similar approach has been proposed by Brenner [9]. Many characteristics and assumptions of the cited methods are close to other macroscopisation methods, such as homogenization theory [6,33]. Indeed, the general agreement between both methods has been described for diffusion problems in [8]. The major features may be summarised in the following terms:

- The macroscopic characteristic scales are supposed to be decoupled from the microscopic ones, each level having its own variable description.

- The Initial Boundary Value Problem (IBVP) that determines the micro-scale fields is solved in an approximated manner in terms of the macro-scale variables and some mapping variables. The approximation is materialised by micro-scale problems or closure problems that completely define these mapping variables.

- Having solved these micro-scale problems, the macroscopic mathematical description is essentially dependent on the estimation of macroscopic coefficients or effective coefficients that are explicitly given in terms of averages of the mapping variables.

One feature of the considered volume averaging method is, therefore, that some additional hypotheses are needed in order to simplify the original problem and relate the micro-scale fields to the macro-scale ones. These additional relations, which we called "closure relations", are problem dependent, and must be consistent with the assumption made of separated scales. This feature is common to almost all homogenisation methods. For example, asymptotic methods are based on regular asymptotic expansions for inner (micro-scale) and outer variables (macro-scale) to be specified, the scaling of which has to be carefully evaluated by order of magnitude analysis of the relevant parameters [21]. Another method involving scales is the time-scale separation between master and slave modes based on centre manifold description [30]. This method has been used to provide a general and rigorous treatment of Taylor dispersion $[20,22,37,4,28,10,31,5]$. This method shares many features with the one examined in this paper, beside a more general background and different objectives. One important starting point for this method is to use steady state solutions as decomposed into a discrete and infinite set of eigenfunctions. Examining a linear 
problem, the temporal solution are then linearly decomposed into those stationary eigenmodes, i.e each stationary eigenmode is associated with a non-stationary one. Among those, the one associated with the trivial zero eigenvalue is called the master mode because it is associated with slow temporal relaxations of interest for long-time asymptotic behaviour. The other temporal modes fulfil fast temporal relaxations whose influence on the master mode can be re-cast into the master equation parameters. The coupling between slave and master modes is obtained from a linear decomposition strictly similar to the above mentioned "closure relations". These closure relation are derived from a Lyapunov-Schmidt reduction $[4,5]$ associated with a small parameter which is the product between the Péclet number and the aspect ratio of the considered tube.

The general philosophy of this master/slave time separation method is then much similar to the one applied in this paper on the spatial level. In the case of volume averaged methods, far-field spatial asymptotic behaviour (sometimes called "fully developed" spatial variations) are interesting in that they describe the evolution of a simple one-dimensional macroscopic field, without requiring of a precise description of supplementary spatial variations. There is nevertheless one major technical difference with the goal pursued in this paper. In the case of the master/slave time separation method, the invariant manifold theorem gives a nice framework for the validity of such slow/fast mode decomposition close to any trivial zero eigenvalue [22] (because the time scales separation is governed by the ratio of the fast to slow modes eigenvalues). This framework can be easily transposed for spatially decaying mode close to a trivial zero eigenvalue [4]. Those zero eigenvalue macroscopic modes might be interesting, especially when the problem has Neumann boundary conditions. In this case, direct Liapounov-Schmidt reduction techniques have been used to assess the convergence of averaging models, for example, when chemical reaction occurs within the fluid [11]. In section 5.1.1 we will compare our results with those obtained in [11] that are re-discussed in the third section of [5]. Those zero eigenvalue macroscopic modes are nevertheless less interesting in transfer problems. In that case they are associated with a spatially uniform eigenmode whose contribution to the transfer between the tube wall and the fluid is zero. Other non-trivial spatially decaying eigenmodes should then be sought. This is especially true when boundary conditions are not of Neumann type, so that there is no trivial zero eigenmode. But, in this case, the invariant manifold theorem hardly guarantees the validity and accuracy of a slow/fast scale decoupling. One of the purposes of this paper is to re-examine the conditions for which a macro/micro decoupling is a sensible approach in the case of a simple convection-diffusion problem, with general boundary conditions. In this sense, the presented analysis extend previous works $[11,5]$ which have used Liapounov-Schmidt reduction techniques close to a 0 eigenmode. Our analysis considers the approximation of non-zero eigenmodes with non-self-adjoint operators. Whilst restricted to a given convection-diffusion problem, this paper examines the precise conditions for which a part of the exact solution can be captured by an averaged model. One important conclusion, for applications purposes, that is drawn from the proposed analysis is that, depending on the chosen averaging method (more precisely depending on the applied weighting function), the non-trivial, interesting eigenmodes can not always be captured. It is therefore of great interest to know better what causes averaging for convection-diffusion problems to work and why.

Moreover, there is an additional interest in our analysis for those willing to use averaged models. Macro-scale equations, as generally introduced in the literature [36], come from first order terms. The "quality" of the first order approximation is often checked through some comparison with direct simulations, or analytical solutions of the micro-scale equations, or by developing estimates for the higher order terms. It is often difficult to have a precise quantitative determination of those terms, and the first approach, if available, is a valuable information. In a preliminary study of the 
tube problem, it was found that the approximation proposed by [29] would provide a reasonable estimate of the exchange term for the established regime in the case of diffusion/advection in a tube with constant temperature or concentration at the surface [15]. The objective of this paper is to exhibit a higher order analysis of the problem from which convergence proofs can be obtained so that a posteriori conditions are found for the definition of the macroscopic scale.

The paper is organised as follows. The second section reviews convection-diffusion problem in the stationary case, and describes its known solutions. A short review of the results obtained with the volume averaging method is also presented in this section to further document the general context of the study. The third section presents a generalisation of the volume averaging method previously used to describe temporal variations $[4,5]$. This leads to a precise formulation of the mathematical convergence to any eigenmode. The fourth section presents the convergence proof in a two-step procedure. Some numerical results associated with the the convergence of different averaging operators are presented at the end of this section.

\section{General background.}

2.1. Convection-diffusion problem. The material exposed in this section closely follows classical steps that may be find in textbooks, see for instance [13]. We first present the dimensionless formulation associated with convection-diffusion of a passive scalar inside a cylinder, with radial coordinate $r$ made dimensionless by the tube radius $R$. This passive scalar could be associated, for instance, with some heat or mass transfer problem, and we will refer to it as $T(r, \phi, z)$. Classicaly, the ratio between convection to diffusion characteristic times is associated with a dimensionless Péclet number $P e=\langle v\rangle R / D_{m}$, where $D_{m}$ is the diffusion coefficient of the passive tracer in the liquid, and $\langle v\rangle$ is the spatially averaged velocity field. The physical problem giving the convection velocity is supposed to be independent of the passive scalar, so that a translation-invariant fully developed flow $v(r)$ settles in the longitudinal direction $z$ along the cylinder principal axis. Making

dimensionless the longitudinal direction $z$ by the tube radius $R$, the stationary governing equation expressing heat - or mass - conservation of the passive scalar $T(r, z)$ reads :

$$
\Delta T=\operatorname{Pev}(r) \partial_{z} T, \quad \text { with } \quad v(r) \geq 0 \quad \text { analytical in } 0,
$$

where $\Delta$ stands for the Laplace operator, which will be appropriately expressed in cylindrical coordinates. As discussed later, we will be mainly interested in situation where $P e \gg 1$. Nevertheless, it is important to note that other definitions of the dimensionless variable in the $z$ direction could be adopted. As a matter of facts, the typical longitudinal variations are linearly increasing with the Péclet number when $P e \gg 1$, and, furthermore, the longitudinal dimensions of the tube could be much larger than its radius. Hence, many authors among which $[4,5]$ prefer to introduce an additional parameter $p e=P e R / L$ where $L$ is some longitudinal characteristic length associated with the axial variations. In this context, many studies such as the classical ones $[35,1]$ have been interested in the limit of $p e \ll 1$, while $P e \gg 1$ so that longitudinal diffusion can be neglected in comparison with transverse diffusion. This choice is important when considering the averaged description of equation (2.1), which should then be written with a small parameter pe instead of a large parameter $\mathrm{Pe}$ on the right hand side. In the following, we will keep using the Péclet number $P e$ parameter for the problem. Of course, this choice should give equivalent results as those obtained from the use of the small parameter pe, as will be explained in section 5.1.1.

In the case of a Newtonian fluid, the velocity field develops a parabolic Poiseuille flow $v(r)=$ $2\left(1-r^{2}\right)$. Because its particular importance, all the numerical results will be given in this case. However all the theoretical results obtained in this paper still hold for general nonnegative velocity 
fields $v(r) \geq 0$ that are analytical in 0 . General velocity profiles are of interest for applications associated with non-Newtonian fluid, such as for example blood for which different analytical model have been proposed for the velocity profile in a tube [14]. This can also be useful in the treatment of turbulent dispersion in tubes, for which the Poiseuille solution is replaced by the turbulent average velocity field, following the double averaging procedure in Pedras and Lemos (2001) [26].

Because of its relevance to many research areas, this partial differential problem has received much attention. Three basic classes of boundary conditions are naturally associated with this cylindrical geometry : adiabatic Neumann boundary condition $\partial_{r} T(r=1, \phi, z)=0$ - we shall refer to it as $\mathcal{N}$ in the following -, constant temperature Dirichlet boundary condition $T(r=1, \phi, z)=0$ - we shall refer to it as $\mathcal{D}$ in the following - or mixed Robin boundary condition $\partial_{r} T(r=1, \phi, z)+\gamma T(r=$ $1, \phi, z)=0$ where $\gamma>0$ may be called Thiele modulus by reference to the case of heterogeneous reaction - we shall refer to it as $\mathcal{R}$ in the following. Furthermore, the passive scalar reference value is chosen so that, far away from the origin, it reaches its equilibrium state, $T(r, \infty)=0$. The only missing boundary condition is the initial value of the scalar field at the cylinder origin $z=0$, $T(r, 0) \equiv T_{0}(r)$, which has to be specified. It is easy to note that the PDE problem (2.1) is not tensorized, so that it does not independently factorise the radial coordinate $r$ and the longitudinal one $z$. Whilst very simple, the linear problem (2.1) does not have any explicit general solution. Hence, many authors have been interested in the special limit for which a variable separation can be found. In the limit of large Péclet number, $P e \gg 1$, when neglecting the longitudinal diffusion compared to the radial one, equation (2.1) degenerates to :

$$
\left(\Delta_{c}+\frac{1}{r^{2}} \partial_{\phi}^{2}\right) T=\operatorname{Pev}(r) \partial_{z} T,
$$

where $\Delta_{c}$ stands for the cylindrical part of the Laplace operator $\Delta_{c} \equiv 1 / r \partial_{r}\left(r \partial_{r}\right)$, and $\phi$ is the azimuthal angle. It can be shown that such an approximation is $O\left(1 / P e^{2}\right)$, because in this limit, the longitudinal typical variations scale linearly with $P e$ [13]. Equation (2.2) associated with either Neumann $\mathcal{N}$, Dirichlet $\mathcal{D}$ or Robin $\mathcal{R}$ boundary conditions, is then a separable problem, for which the PDE degenerates into a Sturm-Liouville ODE problem. Graetz [16] has found that its general solution is associated with the discrete sets $L_{N}, N \in \mathbb{Z}$, of eigenvalues depending on the boundary condition :

$$
T(r, \phi, z)=\sum_{N \in \mathbb{Z}} \sum_{l \in L_{N}} c_{N, l} G_{N, l}(r) e^{i N \phi} e^{\frac{l}{P e} z},
$$

We define the generalised Graetz functions $G_{N, l}$ as the functions of $r$ that satisfy :

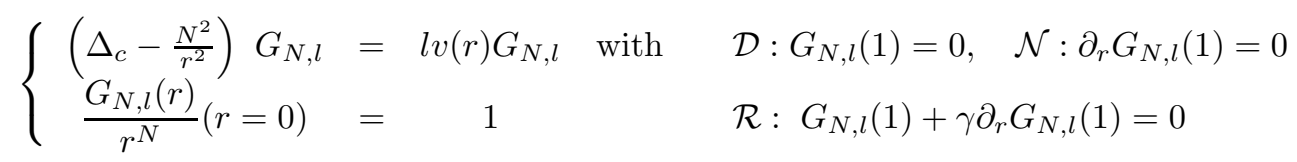

For a general - analytical in 0 - velocity field $v(r)$ one can use the Frobenius method ( $C f$ e.g. [32]) to see that the following equation :

$$
\left(\Delta_{c}-\frac{N^{2}}{r^{2}}\right) y=l v(r) y
$$

which is singular in zero, has two linearly independent solutions $y_{1}$ and $y_{2}$; the first one regular in 0 satisfies $y_{1}(r) / r^{N}(r=0) \neq 0$, and the second one being singular in $0: y_{2}(0)= \pm \infty$. As a result, 
the equation (2.4) with initial condition $G_{N, l} / r^{N}(r=0)=1$ defines a unique function $G_{N, l}$-that we will call generalised Graetz function - for each $l \in \mathbb{C}$ and $N \in \mathbb{Z}$. Thus the following conditions in $(2.4), G_{N, l}(1)=0$ for $\mathcal{D}, \partial_{r} G_{N, l}(1)=0$ for $\mathcal{N}$, or $G_{N, l}(1)+\gamma \partial_{r} G_{N, l}(1)=0$ for $\mathcal{R}$ only selects among these generalised Graetz functions those satisfying the correct boundary condition.

Historically, the cylindrical Graetz functions $G_{0, l}$ has been associated with a parabolic Poiseuille flow $v(r)=2\left(1-r^{2}\right)$ and it is usually found in the literature that the function $G_{0, l}$ is the eigenfunction of $\sqrt{-l}$ rather than $l$. However this notation will be kept for the sake of simplicity in the rest of the paper, and Appendix A gives a more detailed discussion of Graetz eigenfunction and their relations with confluent hyper-geometric functions -or Kummer's functions.

Because (2.4) defines a self-adjoint Sturm-Liouville problem, the eigenvalues associated either with the Dirichlet, Neumann or Robin conditions are real. Moreover, the chosen far-field extinction boundary condition $T(r, \infty)=0$ selects, among those, negative eigenvalues. $L_{N}$ is therefore a discrete set $L_{N} \subset \mathbb{R}^{-}$of ordered eigenvalues $L_{N}=\left\{\cdots<l_{i, N}<\cdots<l_{1, N}<l_{0, N} \leq 0\right\}$. For convenience, we will use a specific notation for the sets associated with Dirichlet, Neumann or Robin boundary conditions, i.e :

$$
\begin{aligned}
L_{N}^{\mathcal{D}}=\left\{l \in \mathbb{R}^{-}, G_{N, l}(1)=0\right\}, & L_{N}^{\mathcal{N}} & =\left\{l \in \mathbb{R}^{-}, \partial_{r} G_{N, l}(1)=0\right\} \\
& \text { or } \quad & L_{N}^{\mathcal{R}}=\left\{l \in \mathbb{R}^{-}, G_{N, l}(1)+\gamma \partial_{r} G_{N, l}(1)=0\right\} .
\end{aligned}
$$

\begin{tabular}{|c|c|c|c|}
\hline$l_{i, N}^{\mathcal{D}}$ & $i=0$ & $i=1$ & $i=2$ \\
\hline$N=0$ & -3.656793458 & -22.30473055 & -56.96051540 \\
\hline$N=1$ & -10.69115115 & -37.38965286 & -80.07477640 \\
\hline$N=2$ & -21.24944651 & -56.05580310 & -106.8036412 \\
\hline$N=3$ & -35.46611328 & -78.38573690 & -137.2070675 \\
\hline \multicolumn{4}{|c|}{ TABLE 2.1} \\
\hline
\end{tabular}

First three elements $(i=0,1,2)$ of sets $L_{N}^{\mathcal{D}}$ for Dirichlet boundary conditions, $N=0,1,2,3$ and a parabolic velocity field $v(r)=2\left(1-r^{2}\right)$

\begin{tabular}{|c|c|c|c|}
\hline$l_{i, N}^{N}$ & $i=0$ & $i=1$ & $i=2$ \\
\hline$N=0$ & 0 & -12.8398060 & -41.93087773 \\
\hline$N=1$ & -4.160532810 & -25.33493287 & -62.48391850 \\
\hline$N=2$ & -12.83980600 & -41.93087773 & -87.08337035 \\
\hline$N=3$ & -26.13743028 & -62.80555035 & -115.8424000 \\
\hline
\end{tabular}

First three elements $(i=0,1,2)$ of sets $L_{N}^{\mathcal{N}}$ for Neumann boundary conditions, $N=0,1,2,3$ and a parabolic velocity field $v(r)=2\left(1-r^{2}\right)$

Graetz has computed the first eigenvalue with two digits precision in [16]. Tables 2.1 and 2.2 give the numerical estimates of the first three eigenvalues associated with a parabolic flow, Dirichlet and Neumann boundary conditions. More complete and precise computations of the eigenvalues can be found, for example, in [34]. S olution given by (2.3) can be completed by the orthogonality 
properties of the eigenfunctions :

$$
\int_{0}^{2 \pi} \int_{0}^{1} G_{N, l}(r) e^{i N \phi} \overline{G_{N^{\prime}, l^{\prime}}(r)} e^{-i N^{\prime} \phi} v(r) r d r d \phi=0 \quad \text { if } \quad N \neq N^{\prime} \text { or } l \neq l^{\prime} .
$$

where the over bar denotes a complex conjugate. Hence, using (2.6), the constant coefficients $c_{N, l}$ in decomposition $(2.3)$ are directly related to the projection of the initial conditions over its corresponding eigenfunction $G_{N, l}$ :

$$
c_{N, l}=\frac{\int_{0}^{2 \pi} \int_{0}^{1} T_{0}(r, \phi) \overline{G_{N, l}(r)} e^{-i N \phi} v(r) r d r d \phi}{2 \pi \int_{0}^{1}\left|G_{N, l}(r)\right|^{2} v(r) r d r} .
$$

Hence, using the eigenfunctions defined in (2.4) the complete solution of the high Péclet limit of the convection-diffusion problem (2.2) within a tube admits a complete spectral representation. Incidentally, the convergence of this representation is known to be rather slow [34]. This is especially true when describing the solution near the origin $z=0$. In this limit, even if (2.3) and (2.7) describe the true mathematical solution, the Lévêque [19] asymptotic expansion should be preferred, because of its simplicity.

Nevertheless, this spectral representation is very useful when only part of the solution is required, as for example, for the far field behaviour when $z>P e /\left(l_{1}-l_{0}\right)$, for which the solution exponentially converges to the first eigenfunction. In the following, we will concentrate on the first eigenfunctions and their associated eigenvalues. We will be furthermore interested in the averaged description of the solution. It should be noted that a uniform averaging along the disk section of the cylinder only keeps axi-symmetrical modes. A more detailed discussion about non-asymmetrical contributions to the averaged description will be discussed in section 5.1.3. The amplitude decomposition (2.7) nevertheless shows that every axi-symmetrical eigenvalue $l_{i, 0}$ contributes to uniformly averaged concentration solution. This should be kept in mind in the following because many results associated with averaged description in the literature have neglected contributions from eigenvalue $l_{i, 0}$, with $i \geq 1$. In the following, we will, for example, see (what is already obvious from directly averaging solution $(2.3) \&(2.7)$ which lead to no contribution of $l \neq 0$ modes for which $\left.\left\langle v(r) G_{N, l}\right\rangle=0\right)$ that a uniform averaging does not permit to capture any decaying eigenvalue associated with the Neumann boundary conditions.

2.2. Weighted volume averaging method. In this section we present an improved version of the volume averaging method introduced in [36] that nevertheless remains closely related to this first method - that we will call "standard volume averaging method". The improvement is based on the introduction of weighted averaging operators as proposed in [15] when the standard volume averaging method only considers averaging associated with the Lebesgue measure. The use of weighted averages had been considered long ago for averaging transport equations [39, 40, $29,12]$. The intentions were to correctly regularize the micro-scale fields, with the objective of improving comparison with experiments. It is interesting to notice that this paper emphasizes another important and fundamental role of weighted averages more related to the mathematical structure of the operator to be averaged.

2.2.1. Definition and notations. To introduce general weighted averaging operators we first introduce the standard averaging operator \langle\rangle corresponding to the Lebesgue measure on each 
cylinder section for functions with radial symmetry :

$$
\langle T\rangle(\phi, z)=2 \int_{0}^{1} T(r, \phi, z) r d r,
$$

and we now define a general weighted averaging operator \langle\rangle$^{\star}$ sometimes simply denoted $\star$ associated with any normalised weight function $w(r)-i . e$. such that $\langle w\rangle=1$ - in cylindrical coordinates as :

$$
\langle T\rangle^{\star}(\phi, z) \equiv T^{\star}(\phi, z)=\langle T w\rangle(\phi, z)=2 \int_{0}^{1} T(r, \phi, z) w(r) r d r .
$$

In the next sections of this paper we will examine special cases of weight function $w$. First, a uniform weight $w=1$ is associated with the standard volume averaging method [36]. Another interesting case, introduced in the preceding section is the "mixing-cup" averaging where the weight function has a dependence exactly similar to that of the velocity field $w(r) \equiv v(r) /\langle v\rangle$. The resulting averaged temperature is also often called bulk temperature. As mentioned in the previous section, this weight function is precisely interesting to be considered in this context because it exactly corresponds to the orthogonalisation operator associated with the Graetz eigenfunctions, as illustrated in (2.6). In the following, the averaging operator is either defined using a specific weight function yet to be specified $w$, or, on the contrary, to simplify the notation, a generic $\star$ is used for averaging (2.8). Now, averaging the theoretical solution (2.3) leads to :

$$
T^{\star}(\phi, z)=\sum_{N \in \mathbb{Z}} \sum_{l \in L_{N}} C_{N, l} e^{i N \phi} e^{\frac{l}{P e} z} \quad \text { with } \quad C_{N, l}=c_{N, l} G_{N, l}^{\star} \in \mathbb{R} .
$$

It should be noted that a supplementary average along the azimuthal direction $\phi$ could be performed. If uniform along $\phi$, such average will only preserve the axi-symmetric mode $N=0$ in (2.9). If the azimuthal averaging is chosen non-uniform along $\phi$, then the averaged solution could have contributions from non axi-symmetric mode $N \neq 0$. In the following, we will be mainly interested in averaging along the radial coordinate. Thus the macroscopic field depends on the azimuthal angle $\phi$. The results that are presented for the convergence of averaging models, will be discussed for any azimuthal mode $N$. Those averaged models, could easily been averaged a second time along $\phi$ to find longitudinally varying averaged equations as finally discussed in subsection 5.1.3.

As mentioned in the introduction, the volume averaging method is a general technique whose purpose is to find a macroscopic description, i.e, an averaged description of a microscopic field that fulfills some PDE problem, without explicitly solving the complete problem, but solving some simplified version of it. Greek letters will be reserved for quantities associated with the volume averaging predictions. Prediction for the scalar field $T$ is thus denoted $\Theta$. In general, the prediction is decomposed into a macroscopic volume averaging prediction $\Theta^{\star}$ and some local deviation $\theta$ to this macroscopic behaviour :

$$
\Theta(r, \phi, z)=\Theta^{\star}(\phi, z)+\theta(r, \phi, z)=\sum_{N \in \mathbb{Z}}\left(\Theta_{N}^{\star}(z)+\theta_{N}(r, z)\right) e^{i N \phi}
$$

with the associated condition $\langle\theta\rangle^{\star}=0$. In the upscaling techniques considered in this paper, the derivation is sought generally under the form of a mapping onto the macroscopic variables and derivatives. The averaged of the microscale equation will be discussed in detail later. This macroscale equation can be used to show that $\Theta^{\star}$ also decomposes into a sum of exponential modes :

$$
\Theta^{\star}(\phi, z)=\sum_{N \in \mathbb{Z}} \Theta_{N}^{\star}(z) e^{i N \phi}
$$


with,

$$
\Theta_{N}^{\star}(z)=\sum_{\lambda \in \Lambda_{N}} C_{N, \lambda} e^{\frac{\lambda}{P e} z} \quad \text { with } \quad C_{N, \lambda}=c_{N, \lambda} \Gamma_{N, \lambda}^{\star} \in \mathbb{R}
$$

where the corresponding Greek letters have been used to describe the approximated discrete spectrum $\Lambda_{N}$ and its corresponding approximated eigenvalues $\lambda$, as well as the corresponding approximated eigenfunction $\Gamma_{N, \lambda}$, approximating $G_{N, l}$, with an approximated amplitude $c_{N, \lambda}$ that will be more explicitly defined in section 4 .

The main purpose of section 4 is to find from which conditions it is possible to find intersections between $\Lambda_{N}$ and the eigenvalue set $L_{N}$ (2.5) of the theoretical problem (2.2). It will be found in section 4.1 that only a part of the spectrum $L_{N}$ can be approximated by elements of $\Lambda_{N}$. It will furthermore be shown in section 4.2 that elements of $\Lambda_{N}$ converges toward these elements of $L_{N}$ that can be approximated when increasing the order of the averaging method. The rate of convergence is consequently studied in section 4.3 .

2.2.2. Weighted volume averaging technique. In this subsection we present the principal steps of the weighted volume averaging technique. The next section will a posteriori justify the classical assumptions made in this section, from examining the weighted volume averaging method generalised to higher order. We will study here both Neumann and Dirichlet Graetz problems. The case of Dirichlet boundary conditions associated with the Graetz problem has been previously examined in the context of the standard volume averaging technique in [15]. The first step of the method is to average the governing equation $(2.2)$, so that $\langle 2.2\rangle^{\star}$ is

$$
P e \partial_{z}\langle v \Theta\rangle^{\star}=\left\langle\Delta_{c} \Theta\right\rangle^{\star}+\frac{1}{r^{2}}\left\langle\partial_{\phi}^{2} \Theta\right\rangle^{\star}=\left\langle\Delta_{c} \Theta\right\rangle^{\star}+\frac{1}{r^{2}} \partial_{\phi}^{2}\langle\Theta\rangle^{\star} .
$$

The next step is to use decomposition (2.10) and (2.11) in (2.13), so that a macroscopic equation is defined for $\Theta_{N}^{\star}$ :

$$
\left\langle\Delta_{c} \Theta_{N}\right\rangle^{\star}-N^{2}\left\langle\frac{\Theta_{N}}{r^{2}}\right\rangle^{\star}=P e \partial_{z}\left\langle v \Theta_{N}\right\rangle^{\star}
$$

The completeness of this macroscopic equation necessitates the knowledge of deviation $\theta_{N}$. The problem associated with the deviation $\theta_{N}$ is now obtained from subtracting (2.14) from (2.2) :

$$
\left(v-\langle v\rangle^{\star}\right) P e \partial_{z} \Theta_{N}^{\star}+P e \partial_{z}\left(v \theta_{N}-\left\langle v \theta_{N}\right\rangle^{\star}\right)=\mathcal{L}_{N}^{\star} \Theta_{N},
$$

where $\mathcal{L}_{N}^{\star}$ stands for the non-local differential operator :

$$
\begin{aligned}
\mathcal{L}_{N}^{\star} \Theta_{N} & =\Delta_{N} \Theta_{N}-\left\langle\Delta_{N} \Theta_{N}\right\rangle^{\star} \\
\Delta_{N} \Theta_{N} & =\Delta_{c} \Theta_{N}-\frac{N^{2}}{r^{2}} \Theta_{N} \\
\left\langle\Delta_{N} \Theta\right\rangle^{\star} & =\left\langle\Delta_{c} \Theta_{N}\right\rangle^{\star}-N^{2}\left\langle\frac{1}{r^{2}} \Theta_{N}\right\rangle^{\star}
\end{aligned}
$$

This operator is neither local nor self-adjoint. It is nevertheless invertible as shown in appendix C. The first term of (2.15) is a macroscopic source term that enters in the microscopic problem defined for deviation $\theta_{N}$. From now on, no hypothesis has been made and the above equations are 
exact. These equations are nevertheless not closed because the coupling between the deviation and the macroscopic field still remains unsolved. Finding this coupling is in fact exactly identical to solving the original problem (2.2), the resolution of which we precisely want to avoid.

Hence, the key step is then to find a suitable hypothesis to close deviation problem (2.14) so that it should only depend on the macroscopic field $\Theta_{N}^{\star}$. First, it should be born in mind that the governing equation (2.2) is linear. As a consequence, it is obvious that the deviation $\theta_{N}$ dependence with the macroscopic field $\Theta_{N}^{\star}$ has to be linear here. Such a linear dependence is in fact very generally admitted in most of the application of the method [36], and comes from the assumption of scale separation. Hence, one writes the "closure hypothesis" by introducing the additional closure field or mapping variables $\alpha_{0,1}(r)$ which relates the deviation $\theta_{N}(r, z)$ to the macroscopic field $\Theta_{N}^{\star}(z)$ :

$$
\theta_{N}(r, z)=\left(w(r) \alpha_{0, N}(r)-1\right) \Theta_{N}^{\star}(z)+w(r) \alpha_{1, N}(r) P e \partial_{z} \Theta_{N}^{\star}(z),
$$

or equivalently :

$$
\Theta_{N}(r, z)=\alpha_{0, N}(r) \Theta_{N}^{\star}(z)+\alpha_{1, N}(r) P e \partial_{z} \Theta_{N}^{\star}(z) .
$$

It is clear that additional terms are required to obtain an exact solution, and this is our objective to understand what has been kept in such an approximate solution. Using the closure hypothesis (2.17) in (2.15) we obtain :

$$
\begin{aligned}
\left(\mathcal{L}_{N}^{\star} \alpha_{0, N}\right) \Theta_{N}^{\star} & +\left(\mathcal{L}_{N}^{\star} \alpha_{1, N}-v(r) \alpha_{0, N}+\left\langle v \alpha_{0, N}\right\rangle^{\star}\right) P e \partial_{z} \Theta_{N}^{\star} \\
& -\left(v(r) \alpha_{1, N}-\left\langle v \alpha_{1, N}\right\rangle^{\star}\right) P e^{2} \partial_{z}^{2} \Theta_{N}^{\star}=0
\end{aligned}
$$

The condition of this equality is that each coefficient multiplying the macroscopic field variations $\Theta^{\star}, \partial_{z} \Theta^{\star}, \partial_{z}^{2} \Theta^{\star}$ are equal to zero. Nevertheless, (2.17) has introduced a closure hypothesis with only two terms, so that, the first two terms should also self-consistently be considered here. This last point is further discussed in the next section. Hence, problems associated with the closure fields $\alpha_{0, N}$ and $\alpha_{1, N}$ are :

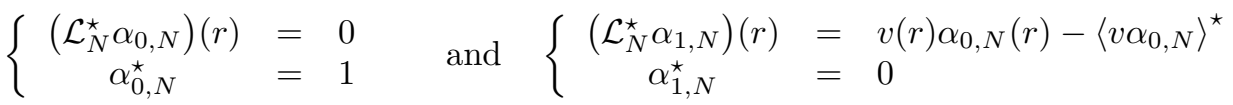

$$
\begin{aligned}
& \text { with } \quad \alpha_{\mathrm{i}, \mathrm{N}}(1)=0 \text { for } \mathcal{D}, \quad \partial_{r} \alpha_{i, N}(1)=0 \text { for } \mathcal{N} \text { or } \\
& \alpha_{i, N}(1)+\gamma \partial_{r} \alpha_{i, N}(1)=0 \text { for } \mathcal{R}, \quad i=1,2
\end{aligned}
$$

These problems can be solved analytically for a Neumann, Dirichlet or Robin boundary condition and their resolution is detailed in appendix $\mathrm{C}$. When introducing these solutions in the macroscopic problem (2.14), one find the following macroscopic problem

$$
K_{0, N} \Theta_{N}^{\star}+K_{1, N} P e \partial_{z} \Theta_{N}^{\star}-\left\langle v \alpha_{1, N}\right\rangle P e^{2} \partial_{z}^{2} \Theta_{N}^{\star}=0
$$

which involves the effective parameters

$$
K_{0, N}=\left\langle\Delta_{N} \alpha_{0, N}\right\rangle^{\star}, \quad K_{1, N}=\left\langle\Delta_{N} \alpha_{1, N}\right\rangle^{\star}-\left\langle v \alpha_{0, N}\right\rangle^{\star},
$$

and the solution for $\Theta_{N}^{\star}$ decomposes to a sum of exponential modes with an associated characteristic length $\mathrm{Pe} / \lambda$ which then defines the set $\Lambda_{1, N}$ of eigenvalues predicted by the volume averaging technique

$$
\Lambda_{1, N}=\left\{\lambda / \quad K_{0, N}+K_{1, N} \lambda-\left\langle v \alpha_{1, N}\right\rangle \lambda^{2}=0\right\} .
$$


2.2.3. Explicit results. This subsection gives the solutions of problem (2.18) i.e, the mapping variables, and (2.19) obtained for different values of the weighted function $w$.

- Standard volume averaging, $w=1$, axi-symmetric mode $N=0$ :

The solution for the closure function has been found equal to :

$$
\text { for } \mathcal{D}:\left\{\begin{array}{l}
\alpha_{0,0}(r)=2\left(1-r^{2}\right) \\
\alpha_{1,0}(r)=\frac{r^{6}}{9}-\frac{r^{4}}{2}+\frac{r^{2}}{2}-\frac{1}{9}
\end{array} \text {, for } \mathcal{N}:\left\{\begin{array}{l}
\alpha_{0,0}(r)=1 \\
\alpha_{1,0}(r)=-\frac{r^{4}}{8}+\frac{r^{2}}{4}-\frac{1}{12}
\end{array}\right.\right.
$$

So that constants $K_{0,0}$ and $K_{1,0}$ can be computed :

$$
\text { for } \mathcal{D}:\left\{\begin{array}{l}
K_{0,0}=-16 \\
K_{1,0}=-2
\end{array}, \text { for } \mathcal{N}:\left\{\begin{array}{l}
K_{0,0}=0 \\
K_{1,0}=-1
\end{array}\right.\right.
$$

These calculations permit to compute the associated approximated eigenvalues by solving (2.21). As already observed in [15], the resulting Dirichlet eigenvalue $\lambda_{0,0}^{\mathcal{D}} \simeq--3.874877690$ gives a rather good approximation of the Graetz value $l_{0,0}^{\mathcal{D}} \simeq-3.656793458$ up to $6 \%$. On the contrary, the Neumann eigenvalue $l_{1}^{N} \simeq-12.839806$ is completely missed by the volume averaging method, which nevertheless gives the trivial solution zero $l_{0,0}^{\mathcal{N}}=0$. This trivial solution is of course of great practical interest since it corresponds to the exact solution when the temperature at the origin is constant, it also gives the correct averaged temperature of the far field solution.

- Flow averaging, $w=v /\langle v\rangle=2\left(1-r^{2}\right)$, axi-symmetric mode $N=0$ :

The solution for the closure function has been found equal to :

$$
\text { for } \mathcal{D}:\left\{\begin{array}{l}
\alpha_{0,0}(r)=\frac{3}{2}\left(1-r^{2}\right) \\
\alpha_{1,0}(r)=\frac{r^{6}}{12}-\frac{3 r^{4}}{8}+\frac{57 r^{2}}{160}-\frac{31}{480}
\end{array}, \text { for } \mathcal{N}:\left\{\begin{array}{l}
\alpha_{0,0}(r)=1 \\
\alpha_{1,0}(r)=-\frac{r^{4}}{8}+\frac{r^{2}}{4}-\frac{1}{16}
\end{array}\right.\right.
$$

So that constants $K_{0,0}, K_{1,0}$ can be computed :

$$
\text { for } \mathcal{D}:\left\{\begin{array}{l}
K_{0,0}=-3 \\
K_{1,0}=-\frac{63}{40}
\end{array}, \text { for } \mathcal{N}:\left\{\begin{array}{l}
K_{0,0}=0 \\
K_{1,0}=-1
\end{array}\right.\right.
$$

The approximate Dirichlet eigenvalue is found in this case equal to $\lambda_{0,0}^{\mathcal{D}} \simeq-3.809523810$ which is $4 \%$ from the theoretical Graetz eigenvalue $l_{0,0}^{\mathcal{D}}$. The Neumann trivial solution $\lambda_{0,0}^{\mathcal{N}}=0$ is also found and the first Neumann non-trivial eigenvalue $\lambda_{1,0}^{\mathcal{N}}$ is also totally missed in the case of a flow averaging.

The following section investigates the capacity of the method to find the correct answer to the problem, while generalising it by introducing higher order closure hypothesis.

3. Weighted volume averaging method of higher order. The notations and methodological steps in this section are closely following those previously presented in sections 2.2 .1 and 2.2.2. More precisely, the solution we are looking for is decomposed as (2.10), and the same exact steps (2.13) to (2.15) are now considered again.

The improvement of the method consists in a generalisation of the closure hypothesis (2.17). This is introduced in order to ameliorate the results previously obtained in section 2.2.3, with, for instance, the hope to capture the first non trivial Neumann eigenvalue $l_{1,0}^{\mathcal{N}}$.

From the property (4.2) of the exact solution, that will be studied in section 4.1.1, and from the previously examined closure relation (2.17) let us now introduce a generalised closure relation :

$$
\Theta_{N}(r, z)=\sum_{n=0}^{p} \alpha_{n, N}(r) P e^{n} \partial_{z}^{n} \Theta_{N}^{\star}(z)
$$


with $p \geq 1$. The case $p=1$ has been analysed in section 2.2.2, and we now follow the same steps. Using (3.1) in the deviation equation (2.15) it is found - assuming that $\alpha_{-1, N}(r)=0$ :

$$
\begin{aligned}
& \sum_{n=0}^{p}\left(\mathcal{L}_{N}^{\star} \alpha_{n, N}-v \alpha_{n-1, N}+\left\langle v \alpha_{n-1, N}\right\rangle^{\star}\right) P e^{n} \partial_{z}^{n} \Theta_{N}^{\star}(z) \\
&-\left(v \alpha_{p, N}-\left\langle v \alpha_{p, N}\right\rangle^{\star}\right) P e^{p+1} \partial_{z}^{p+1} \Theta_{N}^{\star}(z)=0
\end{aligned}
$$

The condition of this equality gives, at each order, the closure problem associated with the closure functions $\alpha_{n, N}(r)$, whose solvability is left to appendix $\mathrm{C}$, and which is to be solved recursively :

$$
\left\{\begin{array}{ccccc}
\mathcal{L}_{N}^{\star} \alpha_{n, N} & = & v(r) \alpha_{n-1, N}(r)-\left\langle v \alpha_{n-1, N}\right\rangle^{\star} & \text { with } & \alpha_{-1, N}(r)=0 \\
\alpha_{0, N}^{\star}=1 & \text { or } & \alpha_{n, N}^{\star}=0 & \text { for } & n \geq 1 \\
\alpha_{n, N}(1) & = & 0 & \text { for } & \mathcal{D} \\
\partial_{r} \alpha_{n, N}(1)= & 0 & \text { for } & \mathcal{N}
\end{array}\right.
$$

The resolution of these problems is detailed in appendix C.2.

From solving (3.2) it is possible to find the generalised macroscopic closed problem at order $p$ :

$$
\sum_{n=0}^{p} K_{n, N} P e^{n} \partial_{z}^{n} \Theta_{N}^{\star}(z)-\left\langle v \alpha_{p, N}\right\rangle^{\star} P e^{p+1} \partial_{z}^{p+1} \Theta_{N}^{\star}(z)=0
$$

where the macroscopic coefficients $K_{n, N}$ are given by :

$$
K_{n, N}=\left\langle\Delta_{N} \alpha_{n, N}\right\rangle^{\star}-\left\langle v \alpha_{n-1, N}\right\rangle^{\star}, \quad K_{n, N} \in \mathbb{R},
$$

The predicted solutions of (3.3) then decompose into a sum of exponentials with modes $\lambda / P e$ for $\lambda$ belonging to the set of predicted eigenvalues at order $p, \Lambda_{p, N}$, defined as the zeros set of a $p+1$ order polynomial :

$$
\Lambda_{p, N}=\left\{\lambda \quad / \quad \sum_{n=0}^{p} K_{n, N} \lambda^{n}-\left\langle v \alpha_{p, N}\right\rangle^{\star} \lambda^{p+1}=0\right\} .
$$

As previously, $\Lambda_{p, N}$ is independent of $P e$, but does depend on the chosen boundary conditions, and the order $p$ of the closure relation. This last point naturally leads to the concept of convergence :

DEFINITION 3.1 (convergence of the weighted volume averaging method).

The elements of all sets $\Lambda_{p, N}$ define sequences of predicted eigenvalues : $\left(\lambda_{i, p}\right)_{p \geq 1, i \geq 0}, \lambda_{i, p} \in \Lambda_{p, N}$. We shall say that the method is convergent toward some eigenvalue $l_{i} \in L_{N}$ of the theoretical problem (2.2) if there exists a sequence of predicted eigenvalues $\left(\lambda_{i, p}\right)_{p \geq 1, i \geq 0}$ such that $\lambda_{i, p} \in \Lambda_{p, N}$ and $\lim _{p \rightarrow+\infty} \lambda_{i, p}=l_{i} \in L_{N}$.

We will establish the convergence for a characterised part of the spectrum in section 4.3.

4. Convergence analysis. Previous sections have mainly considered the explicit application of the averaging method to Graetz problem. The necessary material and notation being now defined, this section considers the mathematical analysis of the convergence of these averaging methods. This convergence analysis requires two different steps. The first step introduces two necessary conditions over eigenvalues, for convergence to hold. The second step gives the proof that these two necessary conditions are sufficient. In the two subsequent sections, the results are derived 
in a general context, and formally apply to any mode $N$, as well as any boundary conditions $\mathcal{D}$, $\mathcal{N}$ or $\mathcal{R}$ and any flow $v(r)$. Hence, in order to simplify notations, the analysis does not mention, unless necessary to avoid confusion, which azimuthal mode it refers to, nor the boundary conditions that is considered. Finally, specific situations will be considered in section 4.3 for analysing the numerical convergence.

4.1. Restricted convergence of weighted averaging methods. We define in the two following subsections two sets, the validity $-D_{v a l}^{\star}-$ and the accessibility $-D_{a c c}^{\star}-$ domains, which are disks lying in the complex plane $\mathbb{C}$. As we will see, a necessary condition for the weighted averaging method to converge toward an eigenvalue $l \in L$ is that this eigenvalue belongs to both these domains.

4.1.1. Validity domain $D_{v a l}^{\star}$. The variables of the initial problem $(2.2)$ can be separated so that any solution $T(r, \phi, z)$ may be written as a product of functions of $r, \phi$ and $z$ only. Let us first show in this section that the exact solution of the problem can be formally written as a regular asymptotic expansion of the macroscopic field $T^{\star}(\phi, z)$. First let us decompose $T$ as :

$$
T(r, \phi, z)=\sum_{N \in \mathbb{Z}} T_{N}(r, z) e^{i N \phi}
$$

The aim of this subsection is to analyse under which condition the $N^{\text {th }}$ component $T_{N}(r, z)$ in decomposition (4.1) of the theoretical solution $T(r, \phi, z)$ can be written as the following expansion of $T_{N}^{\star}(z):$

$$
T_{N}(r, z)=\sum_{n \geq 0} a_{n}(r) P e^{n} \partial_{z}^{n} T_{N}^{\star}(z)
$$

(where the index $N$ on the closure functions $a_{n}(r)$ has been omitted for simplicity) to be compared with the general closure hypothesis (3.1) for $\Theta_{N}(r, z)$.

Let us recall the form of the original solution $(2.3)$ :

$$
T_{N}(r, z)=\sum_{l \in L_{N}} c_{l} T_{l}(r, z) \quad \text { with } \quad c_{l} \in \mathbb{R}, \quad T_{l}(r, z)=G_{N, l}(r) e^{\frac{l}{P e} z}
$$

so that (4.2) is true for $T_{N}(r, z)$ if and only if it holds for each function $T_{l}(r, z)$ standing in the decomposition (4.3) of $T_{N}(r, z)$. Comparing then the expression for $T_{l}$ in $(4.2)$ and (4.3), one can see that (4.2) holds for $T_{l}(r, z)$ if and only if the following equality over the Graetz eigenfunctions $G_{l}$ holds :

$$
\sum_{n \geq 0} a_{n}(r) l^{n}=\frac{G_{N, l}(r)}{G_{N, l}^{\star}}
$$

We will prove that both functions $G_{N, l}(r)$ and $G_{N, l}^{\star}$ are analytical with respect to $l$, so that the expansion of $G_{N, l}(r) / G_{N, l}^{\star}$ on the form (4.4) is only possible for $l$ belonging to a disk $D_{v a l}^{\star}$ centered on zero whose radius $R$ is equal to the smallest root of $G_{l}^{\star}$ :

Definition 4.1. Let us call validity domain the disk $D_{\text {val }}^{\star} \subset \mathbb{C}$ :

$$
D_{v a l}^{\star}=\{l, \quad|l|<R\} \quad \text { where } \quad R=\inf \left\{|l| / \quad G_{N, l}^{\star}=0\right\}
$$


it is depending only on the averaging operator $\star$ and on $N$.

Now, one can see that the decomposition (4.2) is not true in general. It is true only if all the eigenvalues $l$ standing in the decomposition (4.3) of $T_{N}(r, z)$ belong to the validity domain $D_{\text {val }}^{\star}$. An important consequence is that a closure formulation (3.1) only makes sense for eigenvalues lying in $D_{v a l}^{\star}$. Hence, a necessary - but not sufficient - condition for an eigenvalue $l \in L$ to be predicted by the averaging method is to lie within $D_{v a l}^{\star}$. It is also interesting to note that $D_{v a l}^{\star}$ only depends on the averaging operator $\star$, and $N$, but not on the boundary conditions.

We summarise this condition, as well as the definition of the new functions $a_{n}(r)$ in the following lemma (4.2) :

LEMma 4.2. The base functions $T_{l}(r, z)=G_{N, l}(r) e^{\frac{l}{P e} z}$ for problem (2.2) can be written :

$$
T_{l}(r, z)=\sum_{n \geq 0} a_{n}(r) P e^{n} \partial_{z}^{n} T_{l}^{\star}(z)
$$

iff $l \in D_{\text {val }}^{\star}$ defined by

$$
D_{v a l}^{\star}=\{l /|l| \leq R\} \quad \text { where } R=\inf \left\{|l| / G_{N, l}^{\star}=0\right\}
$$

and the functions $a_{n}(r)$ are the solution of the recursive scheme:

$$
\left\{\begin{array}{ccccc}
\Delta_{N} a_{n}(r) & = & v(r) a_{n-1}(r) & \text { with } & a_{-1}(r)=0 \\
a_{0}^{\star}=1 & \text { and } & a_{n}^{\star}=0 & \text { for } & n \geq 1
\end{array}\right.
$$

Proof. In appendix B we give the proof that the functions $G_{N, l}(r), \partial_{r} G_{N, l}(r)$ and $\Delta_{N} G_{N, l}(r)$ are analytical with respect to $l$ on the whole complex plane $\mathbb{C}$. More precisely there exists a set of functions $\left(q_{n}(r)\right)_{n \in \mathbb{N}}$ (depending also on $N$ ) defined by (B.4) such that for $r \in[0,1]$ and $l \in \mathbb{C}$ one has :

$$
G_{N, l}(r)=\sum_{n \geq 0} q_{n}(r) l^{n}, \quad \partial_{r} G_{N, l}(r)=\sum_{n \geq 0} \partial_{r} q_{n}(r) l^{n} \text { and } \quad \Delta_{N} G_{N, l}(r)=\sum_{n \geq 0} \Delta_{N} q_{n}(r) l^{n} .
$$

As a result the three functions $\frac{G_{N, l}(r)}{G_{N, l}^{\star}}, \frac{\partial_{r} G_{N, l}(r)}{G_{N, l}^{\star}}, \frac{\Delta_{N} G_{N, l}(r)}{G_{N, l}^{\star}}$ are analytical with respect to $l$ for $l \in D_{v a l}^{\star}$ and $r \in[0,1]$ and there exist three sets of functions $\left(a_{n}(r)\right)_{n \in \mathbb{N}},\left(b_{n}(r)\right)_{n \in \mathbb{N}}$ and $\left(c_{n}(r)\right)_{n \in \mathbb{N}}$ such that for $l \in D_{v a l}^{\star}$ and $r \in[0,1]$ :

$$
\frac{G_{N, l}}{G_{N, l}^{\star}}(r)=\sum_{n \geq 0} a_{n}(r) l^{n}, \quad \frac{\partial_{r} G_{N, l}}{G_{N, l}^{\star}}(r)=\sum_{n \geq 0} b_{n}(r) l^{n} \text { and } \frac{\Delta_{N} G_{N, l}}{G_{N, l}^{\star}}(r)=\sum_{n \geq 0} c_{n}(r) l^{n}
$$

and using the integration theorem on these series one gets :

$$
b_{n}(r)=\partial_{r} a_{n}(r) \text { and } \quad c_{n}(r)=\Delta_{N} a_{n}(r) \quad \text { for all } n \in \mathbb{N}
$$

so that:

$$
\frac{\Delta_{N} G_{N, l}}{G_{N, l}^{\star}}(r)=\sum_{n \geq 0} \Delta_{N} a_{n}(r) l^{n}
$$


Now the function $\frac{G_{N, l}}{G_{N, l}^{\star}}(r)$ is the unique solution of the ODE :

$$
\Delta_{N} f=l v(r) f \quad \text { and } \quad f^{\star}=1
$$

Rewriting (4.8) with (4.6) and (4.7) gives that the functions $\left(a_{n}(r)\right)_{n \in \mathbb{N}}$ are exactly given by the recursive scheme (4.5).

4.1.2. Accessibility domain $D_{a c c}^{\star}$. The eigenvalues predicted by the averaging method are the roots of the polynomial equation (3.5). Let us consider - as $p \rightarrow \infty$ - the limit set of predicted eigenvalues $\Lambda_{\infty}$ defined as the zeros set of the series $\sum_{n \geq 0} K_{n} \lambda^{n}$ :

$$
\Lambda_{\infty}=\left\{\lambda / \sum_{n \geq 0} K_{n} \lambda^{n}=0\right\}
$$

where the index $N$ on the macroscopic coefficient, assumed to be fixed, has been omitted. Among the eigenvalues predicted with the averaging method at order $p$, the only ones that make sense are these approximating some $\lambda \in \Lambda_{\infty}$, and by increasing the order $p$ of the method one can only improve the computation on these modes $\lambda \in \Lambda_{\infty}$. As a result, for an eigenvalue $l \in L$ of the theoretical problem (2.2) to be approximated by the averaging method, and for this method to be convergent as $p \rightarrow \infty$ to this eigenvalue $l \in L$, it is necessary - but not sufficient - that the series $\sum_{n \geq 0} K_{n} l^{n}$ is convergent.

With definition (3.4) of the macroscopic coefficient $K_{n}$, the series $\sum_{n \geq 0} K_{n} l^{n}$ make sense for $l \in$ $D_{a c c}^{\star}$ defined as follows :

Definition 4.3. Let us call accessibility domain $D_{\text {acc }}^{\star} \subset \mathbb{C}$ the disk of all the complex $\lambda \in \mathbb{C}$ such that the series

$$
\sum_{n \geq 0} \alpha_{n}(r) \lambda^{n} \quad, \quad \sum_{n \geq 0} \Delta_{N} \alpha_{n}(r) \lambda^{n} \quad, \quad \sum_{n \geq 0} \partial_{r} \alpha_{n}(r) \lambda^{n}
$$

are convergent for $r \in[0,1]$. If $\lambda \in D_{a c c}^{\star}$ we say that $\lambda$ is accessible by the averaging method.

On the contrary of the validity domain $D_{v a l}^{\star}$, the accessibility domain $D_{a c c}^{\star}$ does not only depend on the averaging operator $\star$ and on $N$, but also on the boundary conditions that influences the computation of functions $\alpha_{n}$.

4.1.3. Evaluation of $D_{v a l}^{\star}$ and $D_{a c}^{\star}$. We here focus on the numerical evaluation of the two previously introduced domains $D_{v a l}^{\star}$ and $D_{a c}^{\star}$.

To compute the radius of the validity domain $D_{v a l}^{\star}$, we need to compute the smallest root of the function of $l, G_{N, l}^{\star}$. For this, we give in appendix B an expansion of the generalised Graetz functions $G_{N, l}(r)$ with the help of a set of functions $q_{n}(r)$ defined in (B.4) : $G_{N, l}(r)=\sum_{n \geq 0} q_{n}(r) l^{n}$. The computation of these functions $q_{n}(r)$ make possible to compute the radius of $D_{v a l}^{\star}$ as the smallest root of the polynomial $\sum_{n \geq 0} q_{n}^{\star} l^{n}$.

To compute the radius of the accessibility domain $D_{a c c}^{\star}$ one needs an upper bound on the three functions $\alpha_{n}(r), \partial_{r} \alpha_{n}(r)$ and $\Delta_{N} \alpha_{n}(r)$ for $r \in[0,1]$. Experiments based on the computation of these functions showed that $\Delta_{N} \alpha_{n}(r=1)$ is a good upper bound for these functions and the radius of $D_{a c c}^{\star}$ is equal to the convergence radius of the series $\sum_{n \geq 0} \Delta_{N} \alpha_{n}(r=1) \lambda^{n}$.

Radii for $D_{v a l}^{\star}$ and $D_{a c c}^{\star}$ for some chosen weight functions $w(r)$ are given in table 4.1. Comparing table 4.1 with table 2.1 and 2.2 


\begin{tabular}{|c|c|c|c|}
\hline$w(r)$ & $D_{\text {val }}^{\star}$ & $D_{a c c}^{\star}, \mathcal{D}$ & $D_{a c c}^{\star}, \mathcal{N}$ \\
\hline 1 & 7.84 & 15.899 & 10.568 \\
\hline $2\left(1-r^{2}\right)$ & $-l_{1}^{\mathcal{N}} \simeq 12.839$ & 18.632 & 12.839 \\
\hline $1 /(2 r)$ & 354.75 & 24.789 & 14.665 \\
\hline $10(1-r)^{3}$ & $>500$ & 29.82 & 23.33 \\
\hline \multicolumn{4}{|c|}{ TABLE 4.1} \\
Radius of $D_{\text {val }}^{\star}$ and $D_{\text {acc }}^{\star}$ for different weights and for $N=0$
\end{tabular}

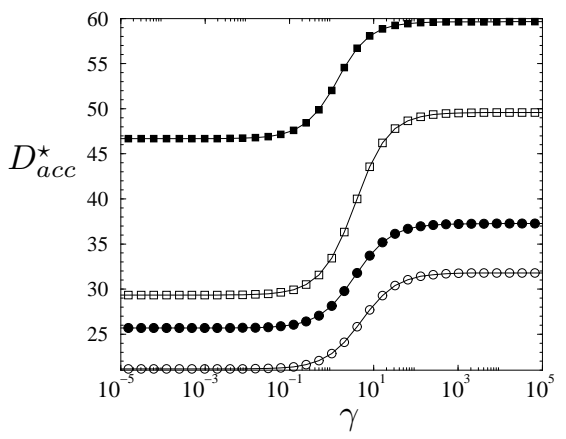

FiguRE 4.1. Computation of the accessibility domain $D_{a c c}^{\star}$ for a Robin boundary condition versus the parameter $\gamma$ and for $N=0$. The four weighting functions $w$ considered on table 4.1 have been analysed. Circular black symbols stands for the flow-averaging method with $w=v /\langle v\rangle$, and circular white symbols for the classical uniform volume averaging $w=1$. White square symbols are for $w=1 /(2 r)$ and black square symbols are for $w=10(1-r)^{3}$.

shows that the standard and the flow averaging method can only capture $l_{0,0}^{\mathcal{D}}$ for $\mathcal{D}$ and $l_{0,0}^{\mathcal{N}}=0$ for $\mathcal{N}$. This result is self-consistent with the computations previously examined in sections 2.2.3. To capture the first non trivial eigenvalue for $\mathcal{N}$ one needs to use other averaging operators. Moreover, it will be shown in section 4.2 that the two necessary conditions introduced in the previous sections are actually sufficient for the convergence to hold. In addition, it will appear that the first non trivial eigenvalue for $\mathcal{N}, l_{1,0}^{\mathcal{N}} \simeq-12.8398060$, and even the second eigenvalue for $\mathcal{D}, l_{1,0}^{\mathcal{D}} \simeq-22.30473055$ can be captured when using adapted averaging operators.

4.2. Convergence theorem. We introduced in the previous subsection two necessary conditions associated with any eigenvalue $l \in L$ to be captured by the averaging method. We prove here that these conditions are actually sufficient for the convergence to hold. More precisely, the eigenvalues predicted by the averaging method when $p \rightarrow \infty$ are exactly the eigenvalues of the theoretical problem (2.2) that both belong to the validity and accessibility domains.

Theorem 4.4. Between the set of eigenvalues $L$ of theoretical problem (2.2) and the three following sets : the validity domain $D_{\text {val }}^{\star}$ defined in definition 4.1, the limit set of predicted eigenvalues $\Lambda_{\infty}$ in (4.9) and the accessibility domain $D_{\text {acc }}^{\star}$ defined in definition 4.3, one has the following relation (for any azimuthal mode $N$, any boundary condition $\mathcal{D}, \mathcal{N}$ or $\mathcal{R}$ and any averaging operator $\star)$ :

$$
\Lambda_{\infty} \cap D_{v a l}^{\star}=L \cap D_{v a l}^{\star} \cap D_{a c c}^{\star},
$$

which means that the eigenvalues predicted by the averaging method inside $D_{\text {val }}^{\star}$ exactly converge toward the theoretical eigenvalues of (2.2) being inside $D_{v a l}^{\star} \cap D_{a c c}^{\star}$. 
Proof. We recall that we defined in (2.4) the generalised Graetz functions $G_{N, l}(r)$ for each $l \in \mathbb{C}$ and each $N \in \mathbb{Z}$ as the unique solution for the ODE :

$$
\Delta_{N} G_{N, l}=l v(r) G_{N, l}(r), \quad \frac{G_{N, l}(r)}{r^{N}}(r=1)=1,
$$

and that, for Neumann, Dirichlet or Robin boundary conditions, the associated sets of theoretical eigenvalues are given by (2.5).

Using lemma 4.2, one has that :

$$
\begin{array}{r}
L^{\mathcal{N}} \cap D_{\text {val }}^{\star}=\left\{l \in \mathbb{C}, \quad \sum_{n \geq 0} \partial_{r} a_{n}(1) l^{n}=0\right\}, \quad L^{\mathcal{D}} \cap D_{\text {val }}^{\star}=\left\{l \in \mathbb{C}, \quad \sum_{n \geq 0} a_{n}(1) l^{n}=0\right\} \quad \text { or } \\
L^{\mathcal{R}} \cap D_{\text {val }}^{\star}=\left\{l \in \mathbb{C}, \quad \sum_{n \geq 0}\left(a_{n}(1)+\gamma \partial_{r} a_{n}(1)\right) l^{n}=0\right\} .
\end{array}
$$

where the functions $\left(a_{n}(r)\right)_{n \in \mathbb{N}}$ are those defined by the recursive scheme (4.5). For simplicity, one introduces the quantities $A_{n}$ defined as :

$$
\text { for } \mathcal{D}: A_{n}=a_{n}(1), \quad \text { for } \mathcal{N}: A_{n}=\partial_{r} a_{n}(1), \quad \text { for } \mathcal{R}: A_{n}=a_{n}(1)+\gamma \partial_{r} a_{n}(1),
$$

so that for $\mathcal{D}, \mathcal{N}$ or $\mathcal{R}$ cases one has

$$
L \cap D_{\text {val }}^{\star}=\left\{l \in \mathbb{C}, \quad \sum_{n \geq 0} A_{n} l^{n}=0\right\} .
$$

Let us consider the two functions of $l$ as the sum of the following series in $l$ :

$$
A_{l}=\sum_{n \geq 0} A_{n} l^{n} \quad, \quad K_{l}=\sum_{n \geq 0} K_{n} l^{n}
$$

which are convergent for $l \in D_{v a l}^{\star} \cap D_{a c c}^{\star}$.

Then, to prove (4.11) one exactly has to show that :

$$
\forall l \in D_{v a l}^{\star} \cap D_{a c c}^{\star} \quad: \quad A_{l}=0 \quad \text { iff } \quad K_{l}=0 .
$$

To prove this, one has to find a relation between $A_{n}$ and the macroscopic coefficient $K_{n}$. For this, one introduces the set of functions $\left(e_{n}(r)\right)$ associated with the difference between functions $a_{n}(r)$ and $\alpha_{n}(r)$ defined in (4.5) and (3.2) :

$$
e_{n}(r)=\alpha_{n}(r)-a_{n}(r) .
$$

These functions, by subtracting (4.5) from (3.2), are exactly defined by the following recursive scheme :

$$
\left\{\begin{array}{ccccc}
\Delta_{N} e_{n}(r) & = & K_{n}+v(r) e_{n-1}(r) & \text { with } & e_{-1}(r)=0 \\
e_{n}^{\star} & = & 0 & & \\
e_{n}(1) & = & -A_{n} & \text { for } & \mathcal{D} \\
\partial_{r} e_{n}(1) & = & -A_{n} & \text { for } & \mathcal{N} \\
e_{n}(1)+\gamma \partial_{r} e_{n}(1) & = & -A_{n} & \text { for } & \mathcal{R}
\end{array}\right.
$$


This recursive formula does depend on both macroscopic coefficients $K_{n}$ and $A_{n}$. Let us finally define the macroscopic difference function $E_{l}(r)$ by :

$$
E_{l}(r)=\sum_{n \geq 0} e_{n}(r) l^{n}=\sum_{n \geq 0} \alpha_{n}(r) l^{n}-\sum_{n \geq 0} a_{n}(r) l^{n},
$$

which is well defined for $l \in D_{v a l}^{\star} \cap D_{a c c}^{\star}$.

We search a differential problem satisfied by $E_{l}(r)$.

Thanks to lemma 4.9 on $D_{v a l}^{\star}$, and to definition 4.3 of $D_{v a l}^{\star}$, the following series :

$$
\begin{array}{lll}
\sum_{n \geq 0} a_{n}(r) l^{n}, & \sum_{n \geq 0} \partial_{r} a_{n}(r) l^{n}, & \sum_{n \geq 0} \Delta_{N} a_{n}(r) l^{n} \text { and } \\
\sum_{n \geq 0} \alpha_{n}(r) l^{n}, & \sum_{n \geq 0} \partial_{r} \alpha_{n}(r) l^{n}, & \sum_{n \geq 0} \Delta_{N} \alpha_{n}(r) l^{n},
\end{array}
$$

converge for all $l \in D_{v a l}^{\star} \cap D_{a c c}^{\star}$ and all $r \in[0,1]$. Then, using the integration theorem and the properties (4.16) of functions $e_{n}(r)$ one has :

$$
\forall l \in D_{\text {val }}^{\star} \cap D_{a c c}^{\star}, \quad \forall r \in[0,1] \quad:\left\{\begin{array}{cccc}
\Delta_{N} E_{l}(r) & = & K_{l}+v(r) E_{l}(r) & \\
E_{l}^{\star} & = & 0 & \\
E_{l}(1) & = & -A_{l} & \text { for } \mathcal{D} \\
\partial_{r} E_{l}(1) & = & -A_{l} & \text { for } \mathcal{N} \\
E_{l}(1)+\gamma \partial_{r} E_{l}(1) & = & -A_{l} & \text { for } \mathcal{R}
\end{array}\right.
$$

$K_{l}$ or $A_{l}$ being fixed, this problem has one and only one solution so that $A_{l}$ is a function of $K_{l}$ and conversely. Now, it is easy to check that the solution associated with $A_{l}=0$ is $E_{l}=0$, which eventually fixes $K_{l}=0$. Conversely, and for the same reason, $K_{l}=0$ fixes $A_{l}=0$. This ensures (4.14), which proves theorem 4.4 .

It is interesting to note that because $E_{l}=0$ is the solution associated with a converging eigenvalue $\lambda_{\infty} \in \Lambda_{\infty}=l \in L$, the ratio between the predicted eigenfunction and its value at $r=0$ also converges to the theoretical Graetz eigenfunction. This leads to the following important corollary :

COROllary 4.5. For an eigenvalue $l \in L \cap D_{\text {val }}^{\star} \cap D_{\text {acc }}^{\star}$, with an associated set of approximated eigenvalues $\left(\lambda_{p}\right)_{p \in \mathbb{N}}$ such that $\lim _{p \rightarrow \infty} \lambda_{p}=$ l, let us define the approximated eigenfunction $\Gamma_{\lambda_{p}}$ as :

$$
\Gamma_{\lambda_{p}}(r)=\frac{1}{\rho} \sum_{n=0}^{p} \alpha_{n}(r) \lambda_{p}^{n}, \text { with } \quad \rho=\sum_{n=0}^{p} \frac{\alpha_{n}(r)}{r^{N}}(r=0) \lambda_{p}^{n},
$$

then $\Gamma_{\lambda_{p}}$ converges toward the generalised Graetz function $G_{N, l}$ :

$$
\lim _{p \rightarrow \infty}\left\|\Gamma_{\lambda_{p}}-G_{N, l}\right\|=0
$$

moreover, defining the amplitude $c_{\lambda}$, in the same way as $c_{l}$ defined in (2.7) :

$$
c_{\lambda_{p}}=\frac{\int_{0}^{2 \pi} \int_{0}^{1} T_{0}(r) \overline{\Gamma_{\lambda_{p}}(r)} e^{-i N \phi} v(r) r d r}{2 \pi \int_{0}^{1}\left|\Gamma_{\lambda_{p}}(r)\right|^{2} v(r) r d r}, \quad \text { then } \lim _{p \rightarrow \infty}\left|c_{\lambda_{p}}-c_{l}\right|=0 .
$$

Hence, not only Theorem (4.4) gives a necessary and sufficient condition for an eigenvalue to converge, but, also, the eigenmode will converge to the corresponding theoretical solution. We now numerically study the convergence of various eigenmodes for different averaging operator $w$. 
4.3. Convergence evaluation. This section studies the numerical evaluation of the convergence to either the eigenvalue, the eigenfunction and the eigenmode amplitude for a Poiseuille parabolic velocity profile $v(r)=2\left(1-r^{2}\right)$. We calculate the closure functions $\alpha_{n}$ from the recursive
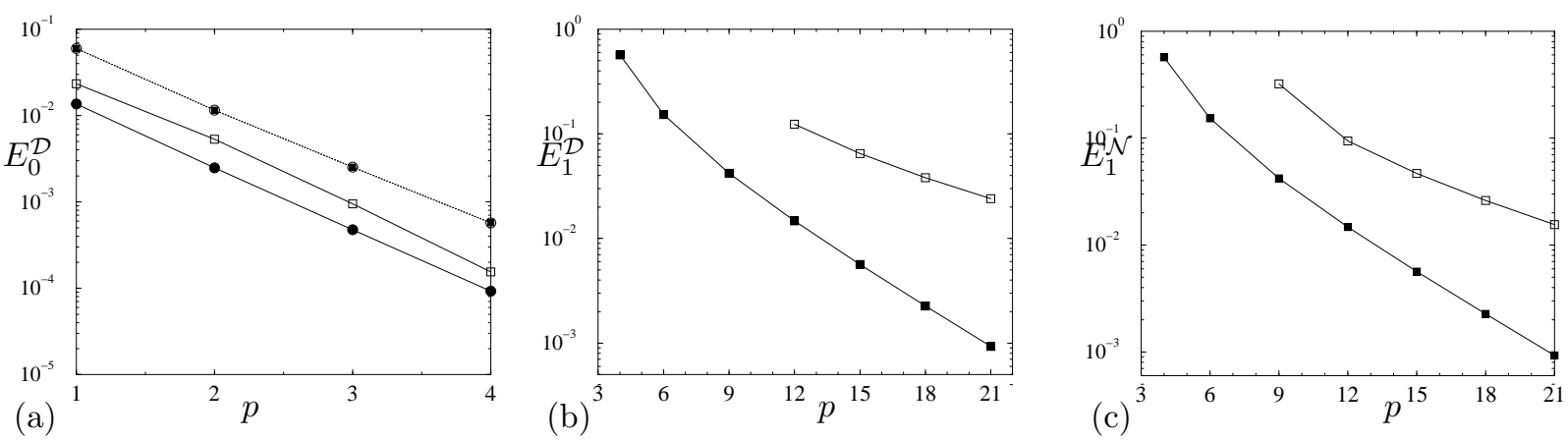

Figure 4.2. Relative error for axi-symmetrical $N=0$ eigenvalues $l_{0,0}$ and $l_{0,1}$ (a) Relative error $E_{0}^{\mathcal{D}}=$ $\left|\lambda_{0, p}^{\mathcal{D}}-l_{0,0}^{\mathcal{D}}\right| / l_{0,0}^{\mathcal{D}}$ between the predicted eigenvalue and the theoretical one $l_{0,0}^{\mathcal{D}}=-3.656793458$, versus the order $p$ of the approximation. Circular black symbols stands for the flow-averaging method with $w=v /\langle v\rangle$, and circular white symbols for the classical uniform volume averaging $w=1$. White square symbols are for $w=1 /(2 r)$ and black square symbols are for $w=10(1-r)^{3}$. In every case the convergence is exponential, as indicated by the observed semi-log linear behaviour. (b) same conventions for the second Dirichlet eigenvalue $l_{0,1}^{\mathcal{D}}=-22.30473055$ convergence $E_{1}^{\mathcal{D}}=\left|\lambda_{1, p}^{\mathcal{D}}-l_{0,1}^{\mathcal{D}}\right| / l_{0,1}^{\mathcal{D}}$. (c) same conventions for the second Neumann eigenvalue convergence $l_{0,1}^{\mathcal{N}}=-12.8398060$ with $E_{1}^{\mathcal{N}}=\left|\lambda_{1, p}^{\mathcal{N}}-l_{0,1}^{\mathcal{N}}\right| / l_{0,1}^{\mathcal{N}}$.
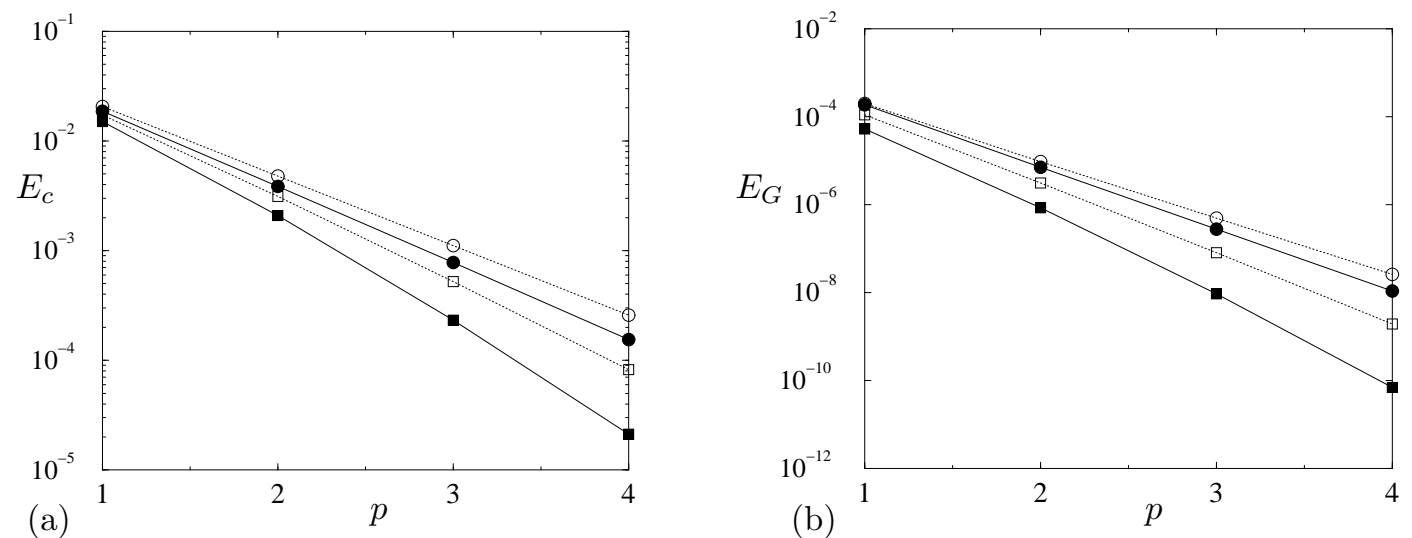

FIGURE 4.3. Relative error for axi-symmetrical $N=0$ eigenmode. (a) Relative error $E_{c}=\left|c_{\lambda_{0, p}^{\mathcal{D}}}-c_{l_{0,0}^{\mathcal{D}}}\right| / c_{l_{0,0}^{\mathcal{D}}}$ between the predicted amplitude and its theoretical value associated with a uniform initial temperature $T_{0}=1$ at $z=0$ for the first Dirichlet eigenmode, versus the order $p$ of the approximation. (b) Absolute error $E_{G}=\left\|\Gamma_{\lambda_{0, p}^{\mathcal{D}}}-G_{l_{0,0}^{\mathcal{D}}}\right\|=$ $\left\langle w\left(\Gamma_{\lambda_{0, p}^{\mathcal{D}}}-G_{l_{0,0}^{\mathcal{D}}}\right)^{2}\right\rangle$ on the predicted eigenfunction for the first Dirichlet eigenmode.

scheme (3.2), so that the coefficients $K_{n}$ defined in (3.4) of the eigenvalues polynomial (3.5) can be computed. From the obtained solution leading to $p+1$ eigenvalues, we select the larger one in $\mathbb{R}^{-}$. Figure 4.2 displays the relative error of this approximated eigenvalue for different weighting 
functions $w$. For the first Dirichlet eigenvalue, figure 4.2(a) displays exponential convergence rates. Moreover, when comparing the results of figure 4.2(a) with table 4.1, it is not surprising to observe that a larger radius of convergence $D_{a c c}^{\star}$ gives rise to a faster convergence rate. As demonstrated in the previous section, the second eigenvalue for Dirichlet or Neumann boundary condition is not accessible to the standard volume averaging methods $-w=1-$ or the kinematic volume averaging $-w=v /\langle v\rangle$ - On the contrary, two other weighting functions $w$ have been proposed in table 4.1, the convergence of which has been established for the second eigenvalue in the previous section. Figures 4.2(b) and 4.2(c) study their convergence on the second eigenvalue in the Dirichlet and Neumann case. It is interesting to observe on these figures that the convergence rate still looks exponential, even if the convergence rate is much slower than those observed on figure 4.2(a). More modes should be indeed needed for an acceptable precision to be obtained.

Moreover, for finite values of the spectral cut-off $p$, the second eigenvalue could not always be captured. For example, this can be observed on Figure $4.2 \mathrm{~b}$ in the case of weighting function $w=10(1-r)^{3}$, for which the eigenvalue becomes real, so that it is considered as being captured by the approximation for $p \geq 12$ only. This example also illustrates that an empirical test of
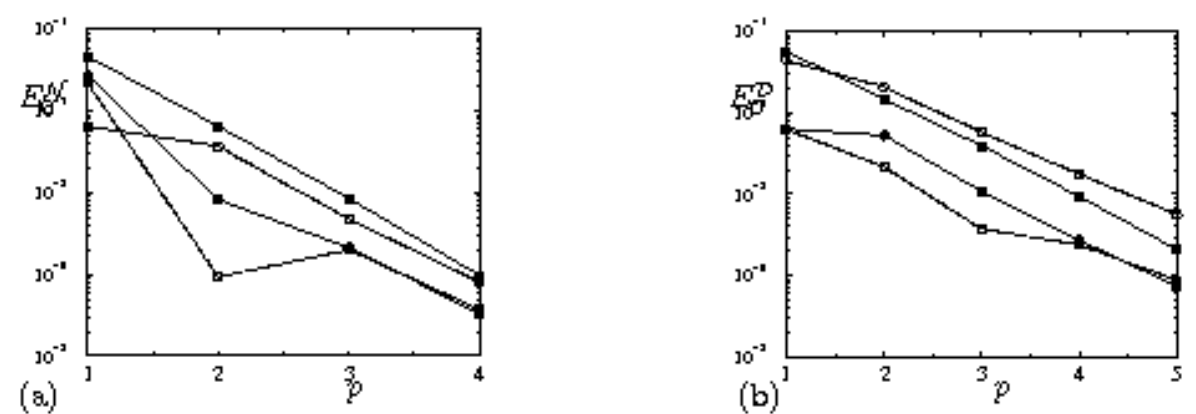

FiguRE 4.4. Same conventions as figure 4.2 for the relative error for non axi-symmetrical $N=1$ eigenvalues $l_{1,0}$ (a) Relative error $E_{0}^{\mathcal{N}}=\left|\lambda_{0, p}^{\mathcal{N}}-l_{1,0}^{\mathcal{N}}\right| / l_{1,0}^{\mathcal{N}}$ between the predicted eigenvalue and the theoretical Neumann one $l_{1,0}^{\mathcal{N}}=-4.160532810$, versus the order $p$ of the approximation. (b) same conventions for the first Dirichlet eigenvalue convergence $l_{1,0}^{\mathcal{D}}=-10.69115115$ with $E_{0}^{\mathcal{D}}=\left|\lambda_{1, p}^{\mathcal{D}}-l_{1,0}^{\mathcal{D}}\right| / l_{1,0}^{\mathcal{D}}$.

the convergence is not always successful. If one would have guessed, ignoring the convergence proof, from the computation of the first ten mapping variables $\alpha_{p}, p<10$ that the first eigenvalue computed in figure $4.2 \mathrm{~b}$ is captured by the weighting function $w=10(1-r)^{3}$, it would have found the wrong answer. Figure 4.3 displays the convergence of the amplitude and the eigenfunction defined in corollary (4.5), for the first Dirichlet mode. It is interesting to note that even the first approximation $p=1$ that has been detailed in section 2.2.3 permits a rather precise amplitude and eigenmode estimate for every tested weighting function $w$. The convergence rate displayed on figure 4.3 is also found to be exponential, as already observed for the eigenvalue convergence. This result does not seem very surprising, for the generalised averaging method has many characteristics in common with a spectral discrete method.

Finally, non-axi-symmetrical mode convergence have been investigated. The convergence of the leading order $N=1$ eigenvalue is represented on figure 4.4. It is interesting to observe that low order approximation (e.g $p<5)$ give rises to a rather precise estimation of this first non-axisymmetric mode. It should then be noted that for both Neumann and Dirichlet boundary conditions, $\left|l_{1,0}\right|$ is smaller than $\left|l_{0,1}\right|$. Hence, the better convergence of figure $4.4 \mathrm{a}$, b compared to $4.2 \mathrm{~b}, \mathrm{c}$ can be 
qualitatively understood. Neumann and Dirichlet situations gives lower and upper bound for the convergence of the more general Robin boundary condition, when varying $\gamma$ from 0 to infinity. Hence, the Robin case should converge the same way as it is observed on the above figures.

\section{Discussion and Conclusion.}

5.1. Discussion. This section discusses the results obtained in the previous sections in the light of previous analysis found in the literature.

5.1.1. Context of the presented analysis. As already mentioned in section 2.1 after defining the convection diffusion problem, (2.1), different characteristic lengths can be chosen for making dimensionless the longitudinal dimension $z$ and this leads to different Péclet number $P e \gg 1$ or $p e \ll 1$. Any choice should lead to consistent results. When choosing the Péclet number $P e \gg 1$, it is known that longitudinal variations along $z$ scale linearly with $P e$. This result holds as an asymptotic expansion discarding $O\left(1 / P e^{2}\right)$ terms [1], and leads to the simplified constitutive equation (2.2). Balakotaiah and Chang[5] mention that the condition $P e \gg 6.93$ is necessary for neglecting axial diffusion. The linear scaling of $z$ variations with $P e$ is described in solution (2.3) and used in the average description of the problem (2.12). From this non-dimensionalisation choice, it appears that standard [36] "ad-hoc" closure relations used in (2.17) and (3.1) do not depends on the Péclet number because each $z$ derivative cancels the corresponding algebraic dependence in $P e$. It then appears that closure relations (2.17) and (3.1) are in fact an asymptotic expansion that involves the eigenvalue $l$ of the problem as a small parameter. The validity range of this asymptotic expansion, that should better be described as an analytic expansion of the eigenfunction with the eigenvalue, is investigated in section 4 , while in the mean time the "ad-hoc" closure relations are $a$ posteriori justified by the convergence proof obtained in the same section. All the validity range result for eigenvalues are obtained independently from the value of the Péclet number, but are valid for $P e \gg 1$ since the starting constitutive equation (2.2) derives from (2.1) discarding $O\left(1 / P e^{2}\right)$ terms [1].

5.1.2. Comparison with other convergence results. It is now interesting to more clearly compare our analysis with previously obtained convergence results. For example, some convergence criteria have been discussed in the context of centre manifold approximations of the convectiondiffusion problem (2.1) by Balakotaiah \& Chang in [4]. In the case of spatially varying solutions, the solution is projected over Graetz eigenfunctions. and a criterion has been found from summing the expansion series. The convergence criterion can be expressed in the same framework from considering the smallest longitudinal variations associated with a critical $\lambda_{c}$. In the case of Dirichlet boundary conditions, it was found $\lambda_{c}^{\mathcal{D}}=13.80$ in [4], whereas $\lambda_{c}^{\mathcal{N}}=37.7$ was obtained in the case of Neumann boundary conditions. These values have to be compared with table 4.1 results. One has to note, that, in our case, the convergence radius $D_{a c c}$ obtained from computing the expansion series, is not the only relevant parameter for convergence. $D_{v a l}$, which comes from the analyticity condition on the averaged Graetz eigenfunction that we wish to approximate, has to be considered also. The convergence radius is the minimum of $D_{a c c}$ and $D_{v a l}$.

Liapounov-Schmidt reduction technique such as used in $[11,5]$ is also another method that should be compared to our analysis. As mentioned in the introduction, this approximation shares much similarities with ours, and the results are also quite similar. In this case, the considered equation (2.1) is written by making dimensionless the longitudinal direction $z$ by $L$ so that the Péclet number is replaced by the small parameter $p e=P e R / L$, as already indicated in section 2.1. The first step of Liapounov-Schmidt reduction approximation is to look for a regular asymptotic 
expansion solution of (2.1) in terms of the small parameter pe. The solution is then decomposed in two parts similar to (2.10) \& (3.1) (but based on a splitting of the linear operator eigenfunctions into "master" eigenfunctions of the kernel of the adjoint operator, and "slave" eigenfunctions of the image of the adjoint operator, see for example [11]). In the case of Neumann boundary condition and weighting function $w=1$, the first closure field solution that we have obtained are exactly similar with those previously obtained in $[11,5]$. More precisely, the first slave mode computed in Eq. (31) of [11] or Eq.(3.8) of [5] corresponds to the Neumann solution $\alpha_{1,0}$ found in (2.22). Nevertheless, higher expansion closure fields differ from those of Chakraborty \& Balakotaiah [11]. From summing the expansion series those authors have been able to find a convergence radius for the approximation. Following criteria (73) of [11], and section 3 discussion of [5], the convergence radius of the Neumann boundary conditions with uniform averaging is $\lambda_{c}^{\mathcal{N}}=48 \times 0.288=13.8$ which should be compared with the value 10.56 of table 4.1 .

This comparison shows that some of our convergence results are very similar with those previously obtained in the literature with other approaches.

5.1.3. Azimuthal averaging. In this section we discuss the possibility and the interest of azimuthal averaging. First, it should be noted that relation (2.7) gives the amplitude of each nonaxisymmetrical mode of the theoretical solution. If, for example, an initial condition is chosen with a pulse at a given location $\left(r_{0}, \phi_{0}\right)$, i.e $T_{0}(r, \phi)=\delta\left(r-r_{0}\right) \delta\left(\phi-\phi_{0}\right)$, then all non-axisymmetrical mode $N \neq 0$ will be represented with a weight $c_{N, l}=G_{N, l}\left(r_{0}\right) v\left(r_{0}\right) r_{0} / \int_{0}^{1}\left|G_{N, l}(r)\right|^{2} v(r) r d r$ because the Fourier transform of the Dirac distribution is uniform. In that case, if one averages the theoretical solution with a uniform weight function along the azimuthal angle $\phi$, all non-axisymmetrical mode $N \neq 0$ will not contribute to the averaged concentration because $\left\langle e^{i N \phi}\right\rangle_{\phi}=0$ for $N \neq 0$. This is not true when using a non uniform averaging operator $w_{\phi}$ along the azimuthal angle $\phi$. In this case, there should be some contribution to the averaged concentration coming from non-axisymmetrical mode $N \neq 0$, summing $c_{N, l}\left\langle e^{i N \phi} w_{\phi}\right\rangle_{\phi}\left\langle G_{N, l}\right\rangle^{\star}$ contributions.

Some of these non-axi-symmetrical contributions to the true averaged concentration solution could be indeed captured by an averaging method, as shown in the previous sections. Hence, for each non-axisymmetrical eigenvalue $l$, one can obtain the appropriate averaging approximation $c_{\lambda_{p}}\left\langle e^{i N \phi} w_{\phi}\right\rangle_{\phi}\left\langle\Gamma_{\lambda_{p}}\right\rangle^{\star}$ of its contribution to the averaged solution.

5.2. Conclusion. This paper analyses the convergence of volume averaging methods on unidirectional convection-diffusion problems. Neumann, Robin and Dirichlet boundary conditions have been considered. The latter problem is of a great interest in the case of local non-equilibrium conditions, i.e, averaged temperature not equal to the value at the boundary, for which approximate solutions are more difficult to obtain.

Concentrating on the stationary solution associated with large Péclet numbers, it has been found that volume averaging methods converge toward the exact solution. A necessary and sufficient condition for this convergence to occur has been found for any unidirectional velocity field, that depends on the averaging operator $w$. This condition has been obtained in a general form, as related to the analytical character of the averaged eigenfunction with the eigenvalue $\lambda$. This condition has in fact a general scope, because it is the basis for writing "closure relations" as a power series of the eigenvalue.

It is interesting to note that the convergence also depends, obviously, on the eigenvalue to be captured. In the case of a parabolic velocity profile, the convergence to the Graetz solution has been studied in more details. In the case of Dirichlet boundary conditions, "natural" operators $w=1$ or $w=v$ allow the convergence to the first non-trivial eigenvalue. In the case of a Neumann 
boundary condition, these usual weighing operators do not capture the first non-trivial eigenvalue of the Graetz problem. In this case, it is necessary to use other averaging operators $w$ to get the first spatially decaying mode, and some of which have been proposed in this paper.

This result shows that averaging over some spatial volume unavoidably degenerates the space of mathematically accessible solutions. Nevertheless, despite smoothing out the small scales -the large eigenvalues- the averaged solution can lead to an asymptotically exact representation of the large scale structure -the small eigenvalues- of the solution. It is expected that this conclusion could be of some general scope when decreasing the dimension number of a problem by averaging along part of its dimensions.

Moreover, the mathematical proof presented in this paper has been complemented in the case of a parabolic Poiseuille flow by some numerical computation of convergence rates. They have been found to be exponential, as expected from a spectral discrete method. It should also be of some general scope, when averaging linear problems. It is interesting to note that the convergence toward non-trivial eigenvalues is directly related to a correct evaluation of the heat transfer between the fluid and the solid boundary. As a matter of fact it should be born in mind that the Nusselt number $N u$, defined as usual as the dimensionless number associated with the heat (or mass) transfer [13] scales asymptotically, when $z \gg P e /\left(l_{1}-l_{0}\right)$, as $N u=l_{1}^{2} / 2$. Hence, convergence toward the eigenvalue of the averaged model is also directly related to a correct evaluation of the asymptotic transfer between the flow and the solid.

Different extensions of this work could be considered. First, a direct transposition of the convergence proof in the case of a plane geometry, with transverse velocity field, should be easily obtained. The quantitative results on the accessibility domain as well as on the convergence accuracy could nevertheless be different in that case. The second extension of interest should be related to more complicated situations associated with a coupling with conduction in some external solid domain.

\section{Appendix A. Graetz functions and Kummer's functions.}

The generalised Graetz functions are the eigenfunctions of the operator $\frac{1}{1-r^{2}} \Delta_{N}$

$$
\frac{1}{1-r^{2}} \Delta_{N} \equiv \frac{1}{1-r^{2}}\left(\partial_{r}^{2}+\frac{1}{r} \partial_{r}-\frac{N^{2}}{r^{2}}\right)
$$

One wants to solve the self-adjoint Sturm-Liouville problem :

$$
\frac{1}{1-r^{2}} \Delta_{N} f=-\ell^{2} f
$$

where we have introduced the positive eigenvalue $\ell^{2}=-l$ to compare to (2.4). Defining a new function $y$, from $f(r)=r^{N} e^{-\frac{\ell}{2} r^{2}} y\left(\ell r^{2}\right), y$ is then a solution of the hyper geometric equation :

$$
z \partial_{z}^{2} y+(1+N-z) \partial_{z} y-\left(\frac{1+N}{2}-\frac{\ell}{4}\right) y=0
$$

In its more general form, the hyper geometric equation reads :

$$
z \partial_{z}^{2} y+(c-z) \partial_{z} y-a y=0
$$

which possesses two solutions called confluent hyper geometric functions, and when $c=1$ : 
- the first one is singular at $z=0$ and is not considered here,

- the other one is regular, convergent and noted $\Phi(a, c, z)$. It is defined by the Kummer's series (with infinite radius of convergence) :

$$
\Phi(a, c, z)=1+\frac{a}{c} z+\frac{a(a+1)}{c(c+1)} \frac{z^{2}}{2}+\ldots+\frac{a \ldots(a+n-1)}{c \ldots(c+n-1)} \frac{z^{n}}{n !}+\ldots
$$

$f$ is proportional to the Graetz function $G_{N, \ell}$

$$
G_{N, \ell}(r)=r^{N} e^{-\ell r^{2} / 2} \Phi\left(\frac{1+N}{2}-\frac{\ell}{4}, 1+N, \ell r^{2}\right)
$$

\section{Appendix B. Analyticity in $l$ of the Graetz functions.}

In this appendix we prove that the generalised Graetz functions defined in (2.4) $G_{N, l}(r)$ are analytical in $l$ on the whole complex field $\mathbb{C}$. More precisely : for the closure functions $q_{N, n}(r)$ defined in (B.4) one has for each $l \in \mathbb{C}$ :

$$
G_{N, l}(r)=\sum_{n \geq 0} q_{N, n}(r) l^{n}, \quad \partial_{r} G_{N, l}(r)=\sum_{n \geq 0} \partial_{r} q_{N, n}(r) l^{n} .
$$

We point out that this result is true for any $N \in \mathbb{Z}$ and for any flow $v(r)$ that is non negative and analytical in 0 .

We shall prove this result in two steps :

- in B.1 we prove that (B.1) is true when $l$ belongs to a disk $D \subset \mathbb{C}$ which we characterise.

- in B.2 we prove that $D=\mathbb{C}$

We firstly recall the following definitions :

For a given value $N \in \mathbb{Z}$ of the axi-symmetric parameter, the operator $\Delta_{N}$ is defined as : $\Delta_{N} \equiv$ $\partial_{r}^{2}+\frac{1}{r} \partial_{r}-\frac{N^{2}}{r^{2}}$, so that $\Delta_{-N}=\Delta_{N}$. Hence we will consider the case $N \geq 0$ only.

The operator $\Delta_{N}$ can be written under a divergence form :

$$
\Delta_{N} f=\frac{1}{r^{N+1}} \partial_{r}\left(r^{2 N+1} \partial_{r}\left(\frac{f}{r^{N}}\right)\right) .
$$

For each $l \in \mathbb{C}$ the Graetz function $G_{l, N}$ is the only one solution for the following ODE :

$$
\left\{\begin{array}{lll}
\Delta_{N} G_{N, l} & =l v(r) G_{N, l}(r) \\
\frac{G_{N, l}}{r^{N}}(0) & = & 1
\end{array} .\right.
$$

We define the set of closure functions $q_{N, n}$, for $n \geq 0$, as follows :

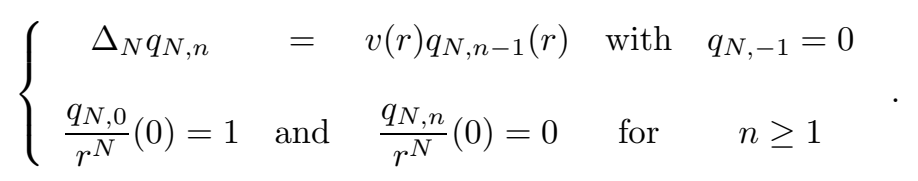




\section{B.1. A criterion for the Graetz function to be analytical in $l$.}

Theorem B.1. Let $D$ be the convergence disk of the series

$$
\sum_{n \geq 0} q_{N, n}(1) l^{n}
$$

where the closure functions $q_{N, n}$ are defined in (B.4), then for all $l \in D$ :

$$
\begin{aligned}
G_{N, l}(r)= & \sum_{n \geq 0} q_{N, n}(r) l^{n}, \quad \partial_{r} G_{N, l}(r)=\sum_{n \geq 0} \partial_{r} q_{N, n}(r) l^{n} \\
& \text { and } \Delta_{N} G_{N, l}(r)=\sum_{n \geq 0} \Delta_{N} q_{N, n}(r) l^{n}
\end{aligned}
$$

Proof. We begin by proving that for a fixed $l \in D$ the three series $\sum_{n \geq 0} q_{N, n}(r) l^{n}, \sum_{n \geq 0} \partial_{r} q_{N, n}(r) l^{n}$ and $\sum_{n \geq 0} \Delta_{N} q_{N, n}(r) l^{n}$ are uniformly convergent for $r \in[0,1]$.

First of all the recursive definition (B.4) of the functions $q_{N, n}$ implies that : for all $n \geq 0$ $q_{N, n}(r)=r^{N} \psi_{n}(r)$ where $\psi_{n}$ is a non negative, non decreasing, continuous function on $[0,1]$ :

$$
q_{N, 0}=r^{N} \quad \text { and } \quad q_{N, n}(r)=r^{N} \int_{0}^{r} \frac{1}{y^{2 N+1}} \int_{0}^{y} x^{N+1} v(x) q_{N, n-1}(x) d x d y \quad \text { for } \quad n \geq 1,
$$

so that $0 \leq q_{N, n}(r) \leq q_{N, n}(1)$ and the series $\sum_{n \geq 0} q_{N, n}(r) l^{n}$ is uniformly converging on $[0,1]$ for $l \in D$.

On the same way $\Delta_{N} q_{N, n}(r)=v(r) q_{N, n-1}(r)$ and so one has $0 \leq \Delta_{N} q_{N, n}(r) \leq\|v\| q_{N, n-1}(1)$ and the series $\sum_{n \geq 0} \Delta_{N} q_{N, n}(r) l^{n}$ is uniformly converging on $[0,1]$ for $l \in D$.

Now one has :

$$
\begin{aligned}
0 \leq \partial_{r} q_{N, n}(r) & =N r^{N-1} \int_{0}^{r} \frac{1}{y^{2 N+1}} \int_{0}^{y} x^{N+1} v(x) q_{N, n-1}(x) d x d y+\frac{1}{r^{N+1}} \int_{0}^{r} x^{N+1} v(x) q_{N, n-1}(x) d x \\
& \leq N r^{N-1} q_{N, n}(1)+\frac{\|v\|}{N+2} r q_{N, n-1}(1) \\
& \leq C\left(q_{N, n}(1)+q_{N, n-1}(1)\right)
\end{aligned}
$$

where the constant $C$ only depends on $N$ and $v$ so that the series $\sum_{n \geq 0} \partial_{r} q_{N, n}(r) l^{n}$ is uniformly converging on $[0,1]$ for $l \in D$.

Now, for a given value $l \in D$ we introduce the two functions defined on $[0,1]$ :

$$
F(r)=\sum_{n \geq 0} q_{N, n}(r) l^{n}, \quad H(r)=\sum_{n \geq 0} \Delta_{N} q_{N, n}(r) l^{n},
$$

since these are uniformly converging series and since $\sum_{n \geq 0} \partial_{r} q_{N, n}(r) l^{n}$ is also a uniformly converging series for $r \in[0,1]$, one can use the integration theorem which implies that :

$$
H(r)=\Delta_{N} F(r), \quad \text { for } r \in[0,1],
$$

and in the same time one has with (B.4) that $H(r)=l v(r) F(r)$ and that $\frac{F}{r^{N}}(0)=1$. The unicity of the solutions of (B.3) ensures then $F(r)=G_{N, l}(r)$ and this ends the proof. 


\section{B.2. Analyticity on the whole complex field $\mathbb{C}$.}

Lemma B.2. The series

$$
\sum_{n \geq 0} q_{N, n}(1) l^{n}
$$

is convergent on the whole complex plane $\mathbb{C}$ and so (B.1) is true for all $l \in \mathbb{C}$.

Proof. With the integral formulation (B.6) on the closure functions $q_{N, n}$ one has:

$$
\begin{aligned}
q_{N, n+m}(1) & =\int_{0}^{1} \frac{1}{y_{1}^{2 N+1}} \int_{0}^{y_{1}} x_{1}^{N+1} v\left(x_{1}\right) q_{N, n+m-1}\left(x_{1}\right) d x_{1} d y_{1} \\
& =\int_{0}^{1} \frac{1}{y_{1}^{2 N+1}} \int_{0}^{y_{1}} x_{1}^{2 N+1} v\left(x_{1}\right) \ldots \int_{0}^{x_{m-1}} \frac{1}{y_{m}^{2 N+1}} \int_{0}^{y_{m}} x_{m}^{N+1} v\left(x_{m}\right) q_{N, n}\left(x_{m}\right) d x_{m} d y_{m} \ldots d x_{1} d y_{1},
\end{aligned}
$$

and, since $0 \leq q_{N, n}(r) \leq r^{N} q_{N, n}(1)$ (see (B.6)), we have :

$$
\begin{aligned}
\frac{q_{N, n+m}(1)}{q_{N, n}(1)} & \leq \int_{0}^{1} \frac{1}{y_{1}^{2 N+1}} \int_{0}^{y_{1}} x_{1}^{2 N+1} v\left(x_{1}\right) \ldots \int_{0}^{x_{m-1}} \frac{1}{y_{m}^{2 N+1}} \int_{0}^{y_{m}} x_{m}^{2 N+1} v\left(x_{m}\right) d x_{m} d y_{m} \ldots d x_{1} d y_{1} \\
& \leq\|v\|^{m} \int_{0}^{1} \frac{1}{y_{1}^{2 N+1}} \int_{0}^{y_{1}} x_{1}^{2 N+1} \ldots \int_{0}^{x_{m-1}} \frac{1}{y_{m}^{2 N+1}} \int_{0}^{y_{m}} x_{m}^{2 N+1} d x_{m} d y_{m} \ldots d x_{1} d y_{1},
\end{aligned}
$$

where $\|v\|=\sup v(r)$.

This upper bound can be computed explicitly

$$
\frac{q_{N, n+m}(1)}{q_{N, n}(1)} \leq\|v\|^{m} \frac{1}{2(2 N+2)} \cdots \frac{1}{2 m(2 N+2 m)}:=\alpha_{m}
$$

and $\alpha_{m}^{-1 / m}$ is a lower bound for the radius of convergence of the series (B.2). One can easily check that:

$$
\alpha^{-1 / m} \geq 2 \frac{2 N+2}{\|v\|}(m !)^{1 / m}
$$

and so $\alpha^{-1 / m}$ grows up to infinity. As a result the series (B.2) is convergent on the whole complex plane $\mathbb{C}$.

\section{Appendix C. Invertibility of the operator $\mathcal{L}_{N}^{\star}$ and resolution of the closure problems.}

In this appendix we prove that the closure problems :

$$
\left\{\begin{array}{ccccc}
\mathcal{L}_{N}^{\star} \alpha_{n} & = & v(r) \alpha_{n-1}(r)-\left\langle v \alpha_{n-1}\right\rangle^{\star} & \text { with } & \alpha_{-1}(r)=0 \\
\alpha_{0}^{\star}=1 & \text { or } & \alpha_{n}^{\star}=0 & \text { for } & n \geq 1 \\
+ \text { boundary condition } & & & &
\end{array}\right.
$$

for a boundary condition either of a homogeneous Dirichlet, homogeneous Neumann or Robin type :

$$
\alpha_{n}(1)=0, \quad \partial \alpha_{n}(1)=0 \text { or } \quad \partial \alpha_{n}(1)+\gamma \alpha_{n}(1)=0,
$$


has one and only one bounded solution for each $n \in \mathbb{N}$.

The operator $\mathcal{L}_{N}^{\star}$ is defined for $N \in \mathbb{Z}$ and for a normalised averaging operator $\star$ (i.e. such that $\langle 1\rangle^{\star}=1$ ) by :

$$
\mathcal{L}_{N}^{\star} f=\Delta_{N} f-\left\langle\Delta_{N} f\right\rangle^{\star}
$$

for the operator $\Delta_{N}$ :

$$
\Delta_{N} f=\partial_{r}^{2} f+\frac{1}{r} \partial_{r} f-\frac{N^{2}}{r^{2}} f .
$$

Because $\Delta_{N}=\Delta_{-N}$ we will only consider here the proof for $N \geq 0$.

We proceed in two steps : in C.1 we prove a lemma on the general solution of $\Delta_{N} f=g$ and in C.2 we apply that lemma to the problems (C.1) for every boundary condition (C.2).

\section{C.1. A technical lemma.}

LEMma C.1. Let $g$ be a continuous function defined on $[0,1]$ and such that $g^{\star}=0$. Then for all $A \in \mathbb{R}$ the $O D E$ :

$$
\begin{aligned}
\Delta_{N} f-A & =g \\
f^{\star} & =M \in \mathbb{R}
\end{aligned}
$$

has one and only one bounded solution on $[0,1]$.

Moreover this solution fulfils :

$$
\left\langle\Delta_{N} f\right\rangle^{\star}=A
$$

and then is a solution of :

$$
\left\{\begin{array}{c}
\mathcal{L}_{N}^{\star} f=g \\
f^{\star}=M
\end{array}\right.
$$

Proof. We define the function $\psi_{1}(r)$ :

$$
\psi_{1}(r)=-r^{N} \int_{r}^{1} \frac{1}{y^{2 N+1}} \int_{0}^{y} x^{N+1} g(x) d x d y
$$

which is well defined since $g$ is continuous in 0 for $N \geq 0$, and the function $\psi_{2}(r)$ :

$$
\psi_{2}(r)=\frac{r^{N}-r^{2}}{N^{2}-4} \quad \text { if } N \neq 2 \text { and } \quad \psi_{2}(r)=\frac{r^{2}}{4} \ln (r) \quad \text { for } N=2 .
$$

Any solution of (C.4) is of the form :

$$
\begin{aligned}
& f(r)=\lambda r^{N}+\mu r^{-N}+A \psi_{2}(r)+\psi_{1}(r) \quad \text { if } N \neq 0 \text { or } \\
& f(r)=\lambda r^{N}+\mu \ln (r)+A \psi_{2}(r)+\psi_{1}(r) \quad \text { if } N=0 .
\end{aligned}
$$


Then all bounded solution of (C.4) on $[0,1]$ are on the form :

$$
f(r)=\lambda r^{N}+A \psi_{2}(r)+\psi_{1}(r),
$$

and (C.5) gives :

$$
\lambda=\frac{M-A \psi_{2}^{\star}-\psi_{1}^{\star}}{\left\langle r^{N}\right\rangle^{\star}}
$$

and so (C.4) (C.5) has only one bounded solution.

Since $g^{\star}=0$ one also has $\left\langle r^{N}\right\rangle^{\star}=A \mathbf{\square}$

\section{C.2. Resolution of the closure problems.}

- Homogeneous Dirichlet case.

We consider the solution $f$ as in (C.8) of (C.4) (C.5) and search a value of $A$ such that $f(1)=0$. We have :

$$
f(1)=\frac{M-A \psi_{2}^{\star}-\psi_{1}^{\star}}{\left\langle r^{N}\right\rangle^{\star}}
$$

So there is only one bounded solution $f$ of (C.4) (C.5) such that $f(1)=0$, it is defined as :

$$
\begin{aligned}
f(r) & =\frac{M-A \psi_{2}^{\star}-\psi_{1}^{\star}}{\left\langle r^{N}\right\rangle^{\star}} r^{N}+A \psi_{2}(r)+\psi_{1}(r) \\
A & =\frac{M-\psi_{1}^{\star}}{\psi_{2}^{\star}},
\end{aligned}
$$

and $A$ is well defined because $\psi_{2}$ is negative and so $\psi_{2}^{\star} \neq 0$.

Consequently, the closure problems (C.1) for an homogeneous Dirichlet boundary condition are well posed.

- Homogeneous Neumann case.

We consider the solution $f$ as in (C.8) of (C.4) (C.5) and search a value of $A$ such that $\partial_{r} f(1)=0$. By multiplying (C.4) by $r^{N+1}$ and integrating over $[0,1]$ one gets :

$$
\partial_{r} f(1)=N f(1)+\frac{A}{N+2}+\int_{0}^{1} r^{N+1} g(r) d r
$$

and since

$$
f(1)=\frac{M-A \psi_{2}^{\star}-\psi_{1}^{\star}}{\left\langle r^{N}\right\rangle^{\star}}
$$

there is only one solution defined as :

$$
\begin{aligned}
f(r) & =\frac{M-A \psi_{2}^{\star}-\psi_{1}^{\star}}{\left\langle r^{N}\right\rangle^{\star}} r^{N}+A \psi_{2}(r)+\psi_{1}(r) \\
A\left(\frac{1}{N+2}-N \frac{\psi_{2}^{\star}}{\left\langle r^{N}\right\rangle^{\star}}\right) & =N \frac{\psi_{1}^{\star}-M}{\left\langle r^{N}\right\rangle^{\star}}-\int_{0}^{1} r^{N+1} g(r) d r,
\end{aligned}
$$


where $\mathrm{A}$ is well defined because $\psi_{2}$ is negative and so $\frac{1}{N+2}-N \frac{\psi_{2}^{\star}}{\left\langle r^{N}\right\rangle^{\star}} \neq 0$.

Consequently the closure problems (C.1) for an homogeneous Neumann boundary condition are well posed.

- Robin case.

We consider the solution $f$ as in (C.8) of (C.4) (C.5) and search a value of $A$ such that $\partial_{r} f(1)+$ $\gamma f(1)=0$ for $\gamma>0$.

With (C.9) and (C.10) we have :

$$
\partial_{r} f(1)+\gamma f(1)=A\left(\frac{1}{N+2}-(N+\gamma) \frac{\psi_{2}^{\star}}{\left\langle r^{N}\right\rangle^{\star}}\right)-(N+\gamma) \frac{\psi_{1}^{\star}-M}{\left\langle r^{N}\right\rangle^{\star}}+\int_{0}^{1} r^{N+1} g(r) d r,
$$

and so there is only one solution defined as :

$$
\begin{aligned}
f(r) & =\frac{M-A \psi_{2}^{\star}-\psi_{1}^{\star}}{\left\langle r^{N}\right\rangle^{\star}} r^{N}+A \psi_{2}(r)+\psi_{1}(r) \\
A\left(\frac{1}{N+2}-(N+\gamma) \frac{\psi_{2}^{\star}}{\left\langle r^{N}\right\rangle^{\star}}\right) & =(N+\gamma) \frac{\psi_{1}^{\star}-M}{\left\langle r^{N}\right\rangle^{\star}}-\int_{0}^{1} r^{N+1} g(r) d r,
\end{aligned}
$$

where $\mathrm{A}$ is well defined for $\gamma \geq 0$.

Consequently the closure problems (C.1) for an homogeneous Neumann boundary condition are well posed.

\section{REFERENCES}

[1] R. ARIS, On the dispersion of a solute in a fluid flowing through a tube, Proc. Roy. Soc. A, 235 (1956), pp. 65-77.

[2] H. Arkin, L. X. Xu, and K. R. Holmes, Recent developments in modeling heat transfer in blood perfused tissues, IEEE Trans. Biomed. Eng., 41 (1994), pp. 97-107.

[3] J. W. Baish, P. S. Ayyaswamy, and K. R. Foster., Heat transport mechanisms in vascular tissues: a model comparison., J. Biomech. Eng., 108 (1986), pp. 324-331.

[4] V. Balakotaiah and H. C. Chang, Dispersion of chemical solutes in chromatographs and reactors, Proc. Trans. R. Soc. Lond. A., 351 (1995), pp. 39-75.

[5] — Hyperbolic homogenized models for thermal and solutal dispersion, SIAM J. Applied Math., 63 (2003), pp. 1231-1258.

[6] J. Bensoussan, L. Lions, and G. Papanicolaou, Asymptotic Analysis for Periodic Structure, North-Holland, Amsterdam, 1978.

[7] A. Berman, Laminar flow in channels with porous walls, Journal of Applied Physics, 24 (1953), pp. $1232-1235$.

[8] A. Bourgeat, M. Quintard, ANd S. Whitaker, Elments de comparaison entre la mthode d'homognisation et la mthode de prise de moyenne avec fermeture, C. R. Acad. Sci. Paris 2, 306 (1988), pp. 463-466.

[9] H. Brenner, Dispersion resulting from flow through spatially periodic porous media, Trans Royal Soc., 297 (1980), pp. 81-133.

[10] M. D. Bryden And H. Brenner, Multiple-timescale analysis of taylor dispersion in converging and diverging flows, J. Fluid Mech., 311 (1996), pp. 343 - 359.

[11] S. Chakraborty and V. Balakotaiah, Low-dimensional models for describing mixing effects in laminar flow turbulent reactors, Chemical Engineering Science, 57 (2002), pp. 2545-2564.

[12] J. H. Cushman, L. S. Bennethum, And B. X. Hu, A primer on upscaling tools for porous media, Advances in Water Resources, 25 (2002), pp. 1043-1067.

[13] W. Deen, Analysis of transport phenomena, Oxford University press, 1947.

[14] Y. C. Fung, Biomechanics : mechanical properties of living tissues, Springer, Second edition, 1996.

[15] F. Golfier, M. Quintard, And S. Witaker, Heat and mass transfer in tubes : an analysis using the method of volume averaging, Journal of Porous Media, 5 (2002), pp. $169-185$.

[16] L. Graetz, On the thermal conductivity of liquids, Ann. Phys. Chem., 18 (1883), pp. 79-94. 
[17] S. Hassanizadeh ANd W. Gray, General conservation equations for multi-phase systems 1.averaging procedure, Advances in Water Resources, 2 (1979), pp. 131-144.

[18] S. Kakac, R. K. Shah, And A. E. Bergles, Low Reynolds number flow heat exchangers, Hemisphere publishing corporation, 1983.

[19] M. A. LÉvÊQue, Les lois de transmission de la chaleur par convection, Annales des Mines, Paris, 13 (1928), pp. 201-409.

[20] E. M. Lungu And H. K. Moffat, The effect of wall conductance on heat diffusion in duct flow, J. Engng. Math., 16 (1982), pp. 121-136.

[21] C. Mei, J. L. Auriault, And C. NG, Some applications of the homogenization theory, Advances in Applied Mechanics, 32 (1996), pp. 278-348.

[22] G. N. Mercer And A. J. Roberts, A center manifold description of contaminant dispersion in channels with varying flow properties, SIAM J. Appl. Math, 50 (1990), pp. 1547-1565.

[23] A. Nakayama, F. Kuwahara, A. NaOki, and G. XU, A volume averaging theory and its sub-control-volume model for analyzing heat and fluid flow within complex heat transfer equipment, in 12th International Heat Transfer Conference, J. Taine, ed., vol. 2, Grenoble, 2002, Elsevier, Paris, pp. 851-856.

[24] A. Nakayama, F. Kuwahara, M. Sugiyama, and G. Xu, A two-energy equation model for conduction and convection in porous media, International Journal of Heat and Mass Transfer, 44 (2001), pp. 4375-4379.

[25] D. A. Nelson, Invited editorial on "pennes' 1948 paper revisited", J Appl Physiol, 85 (1998), pp. 2-3.

[26] M. Pedras And M. D. Lemos, Macroscopic turbulence modeling for incompressible flow through undeformable porous media, Int. J. Heat and Mass Transfer, 44 (2001), pp. 1081-1093.

[27] H. H. Pennes, Analysis of tissue and arterial blood temperatures in the resting human forearm., J. Appl. Physiol., 1 (1948), pp. 93-122.

[28] C. G. Phillips, S. R. Kaye, and C. D. Robinson, Time-dependent transport by convection and diffusion with exchange between two phases, J. Fluid Mech., 297 (1995), pp. 373 - 401.

[29] M. Quintard and S. Whitaker, Convection, dispersion, and interfacial transport of contaminants: Homogeneous porous media, Advances in Water resources, 17 (1994), pp. 221-239.

[30] A. J. RoberTs, The utility of an invariant manifold description of the evolution of a dynamical system, SIAM J. Appl. Math., 20 (1989), pp. 1447-1458.

[31] S. Rosencrans, Taylor dispersion in curved channels, SIAM J. Appl. Math., 57 (1997), pp. 1216-1241.

[32] S. L. Ross, Differential equations, A Blaisdell Book in the Pure and Applied Sciences, London, 1964.

[33] E. Sanchez-Palencia, Non-homogeneous media and vibration theory, vol. 127, Springer, New York, 1980.

[34] R. K. Shah And A. L. London, Laminar flow forced convection in ducts, supplement 1 to advances in Heat transfer, academic press, New-York (1978).

[35] G. I. TAYLOR, Dispersion of solute matter in solvant flowing slowly through a tube, Proc. Roy. Soc. A., 219 (1953), pp. 186-203.

[36] S. WhitakeR, The method of volume averaging, Kluwer Academic Punlishers, 1999.

[37] W. R. Young And S. Jones, Shear dispersion, Physics of Fluid, 3 (1991), pp. 1087-1101.

[38] Z.-G. Yuan, W. H. Somerton, and K. S. Udell, Thermal dispersion in thick-walled tubes as a model of porous media, Int. J. Heat Mass Transfer, 34 (1991), pp. 2715-2726.

[39] C. M. MARLE, On macroscopic equations governing multiphase flow with diffusion and chemical reactions in porous media, Internat. J. Engrg. Sci., 20 (1982), pp. 642-682.

[40] J. H. Cushman, Unifying the Concepts of Scale, Instrumentation, and Stochastics in the Development of Multiphase Transport Theory, Water. Res. Resour., 20 (1984), pp. 1668-1676. 